\title{
2'-O-methylation alters the RNA secondary structural ensemble
}

Hala Abou Assi, ${ }^{1,2}$ Honglue Shi, ${ }^{3}$ Bei Liu, ${ }^{1}$ Mary C. Clay, ${ }^{1}$ Kevin Erharter,${ }^{4}$ Christopher Kreutz, ${ }^{4}$ Christopher L. Holley, ${ }^{2^{*}}$ and Hashim M. Al-Hashimi ${ }^{1,3 *}$

1Department of Biochemistry, Duke University School of Medicine, Durham, NC 27710, USA

2Department of Medicine, Duke University School of Medicine, Durham, NC 27710, USA

${ }^{3}$ Department of Chemistry, Duke University, Durham, NC 27708, USA

${ }^{4}$ Institute of Organic Chemistry and Center for Molecular Biosciences Innsbruck (CMBI), University of Innsbruck, 6020 Innsbruck, Austria

* To whom correspondence should be addressed. Tel: +1 919668 2688; Fax: +1 919613 2321;

Email: cholley@duke.edu and Tel: +1 919660 1113; Fax: +1 919684 8885; Email:

hashim.al.hashimi@duke.edu

Present Address: Mary C. Clay, Department of Structural Biology, St. Jude Children's Research Hospital, Memphis, TN, USA 


\section{ABSTRACT}

2'-O-methyl $(\mathrm{Nm})$ is a highly abundant post-transcriptional RNA modification that plays important biological roles through mechanisms that are not entirely understood. There is evidence that $\mathrm{Nm}$ can alter the biological activities of RNAs by biasing the ribose sugar pucker equilibrium toward the C3'endo conformation formed in canonical duplexes. However, little is known about how Nm might more broadly alter the dynamic ensembles of non-canonical RNA motifs. Here, using NMR and the HIV-1 transactivation response (TAR) element as a model system, we show that $\mathrm{Nm}$ preferentially stabilizes alternative secondary structures in which the $\mathrm{Nm}$-modified nucleotides are paired, increasing both the abundance and lifetime of a low-populated short-lived excited state by up to 10 -fold. The extent of stabilization increased with number of $\mathrm{Nm}$ modifications and was also dependent on $\mathrm{Mg}^{2+}$. Through phi $(\Phi)$ value analysis, the $\mathrm{Nm}$ modification also provided rare insights into the structure of the transition state for conformational exchange. Our results suggest that $\mathrm{Nm}$ could alter the biological activities of Nm-modified RNAs by modulating their secondary structural ensembles as well as establish the utility of $\mathrm{Nm}$ as a tool for the discovery and characterization of RNA excited state conformations. 


\section{INTRODUCTION}

$\mathrm{Nm}$ is a highly abundant post-transcriptional modification (Figure $1 \mathrm{~A})$ found in non-coding (1-3) and coding RNAs $(4,5)$. It can be added to the ribose sugar moiety of all four nucleotides $(\mathrm{Nm}=\mathrm{Am}$, $\mathrm{Um}, \mathrm{Cm}$ and $\mathrm{Gm}$ ) (Figure $1 \mathrm{~A}$ ) either via stand-alone methyltransferases (6) or by the enzyme fibrillarin when guided by box C/D small nucleolar RNAs (snoRNAs) $(7,8)$. Nm modifications are important for the cellular activity of diverse RNAs $(9,10)$ and have been linked to diseases (11-14). For example, ribosomal RNAs (rRNA) contain more than $100 \mathrm{Nm}$ sites (15), many in the decoding and peptidyltransferase centers (16). Downregulation of fibrillarin and loss of rRNA Nm sites results in impaired ribosomes that are incapable of translating mRNA (17). Conversely, overexpression of fibrillarin and the concomitant increase in $\mathrm{Nm}$ modifications is associated with an increase in protein translation in rapidly dividing breast cancer cells (12). In the spliceosome, all small nuclear RNAs (snRNAs) harbor $\mathrm{Nm}$ modifications, some of which are required for proper spliceosome assembly and function $(18,19)$. The loss of Nm modifications on snRNA U6 due to knockout of the La-related protein, which helps load U6 snRNA onto the box C/D snoRNA protein complex (20), changes splicing fidelity, impairs spermatogenesis in mice (20), and contributes to the Alazami syndrome (21). Loss of snoRNA-guided $\mathrm{Nm}$ modifications on snRNAs also leads to profound defects in cardiac mRNA splicing and development $(13,22,23)$.

In contrast to the well-studied post-transcriptional modification $N^{6}$-methyladenosine $\left(\mathrm{m}^{6} \mathrm{~A}\right)(24-$ 28), which mainly exerts its biological activity by recruiting reader proteins (28-31), no reader proteins have been discovered for $\mathrm{Nm}$ to date. Rather, it appears that in some cases, the $\mathrm{Nm}$ modification serves to alter the conformational preferences of the Nm-modified RNAs. In isolated mononucleotides and unpaired pyrimidine nucleotides, the ribose moiety exists in a 60:40 dynamic equilibrium between C3'-endo and C2'-endo sugar pucker conformations (32), whereas most paired nucleotides or nucleotides that stack intra-helically predominantly adopt the C3'-endo conformation (33). In pyrimidine nucleosides $(\mathrm{Um}$ and $\mathrm{Cm})$ and dinucleotides (UmpU and $\mathrm{CmpC}), 2$ '-O-methylation biases the sugar pucker equilibrium in favor of C3'-endo by $0.1-0.6 \mathrm{kcal} / \mathrm{mol}$ (Figure 1B) (34-37). This bias arises due to intra-residue steric repulsion between the 2'-O-methyl, the 3'-phosphate, and the 2-carbonyl groups when in the C2'-endo conformation (34). The Nm modification has also been shown to stabilize RNA helices by an average of $\sim 0.2 \mathrm{kcal} / \mathrm{mol}$ per $\mathrm{Nm}$ (38). This is due to a reduction in entropy loss upon duplex formation, as a result of pre-organizing the C3'-endo sugar pucker in the single-strand $(34,39)$ 
and/or favorable enthalpic interactions (40). Since $\mathrm{Nm}$ affords increased thermal stability of duplexes and greater stability toward nuclease digestion, it has important applications in FDA-approved oligonucleotide therapeutics (41-44), siRNAs $(45,46)$, and anti-miRNAs $(47)$.

These biases in RNA conformation induced by Nm can serve important biological roles. For example, the bias in sugar pucker introduced by Um at the first anticodon position in tRNA is proposed to reduce misrecognition of noncognate codons, which require a C2'-endo conformation (34). In the A loop of the 23S rRNA, the universally conserved Um2552 intercalates between two bases to help maintain the active conformation for the $\mathrm{G} 2553$ base, which is directly involved in accommodation of the aminoacyl-tRNA (48). These and other modifications appear to play the role of maintaining distorted RNA conformations at the functional interfaces of the ribosome (48). U2-U6 and U4-U6 snRNAs are critical structural elements of the spliceosome that are rich in Nm modifications (3). These snRNAs undergo changes in secondary structure and base-pairing which are essential for the assembly and disassembly of the spliceosome as well as in cycling between different conformational states required for catalysis $(49,50)$. Nm modifications in the active core of the spliceosome U2-U6 snRNA complex have been shown to promote a conformational transition between a three-way and four-way junction proposed to aid correct positioning of pre-mRNA for exon ligation (51). The modification can also sterically hinder stabilizing interactions between $\mathrm{Nm}$ modified mRNAs and ribosomal-monitoring bases, thus disrupting tRNA selection and proofreading and inhibiting translation $(5,52)$.

For the vast majority of the cases however, the role of $\mathrm{Nm}$ modifications has remained elusive. While it is known that $\mathrm{Nm}$ modifications can stabilize canonical duplexes, little is known about how the modification might more broadly impact the propensities of RNAs to form alternative secondary structures in more complex motifs (such as U2-U6) in which they are frequently found $(10,53,54)$. By biasing sugar pucker and favoring helical conformations, we hypothesized that $\mathrm{Nm}$ could preferentially stabilize alternative secondary structures in which the $\mathrm{Nm}$-modified residues are helical with predominantly C3'-endo sugar pucker, relative to conformations in which the $\mathrm{Nm}$-modified residues are non-helical and enriched in the C2'-endo sugar. These alternative secondary structures, often referred to as 'excited states' (ESs) (55-62), are typically transient and low-abundance, but they have been shown to play important roles in the biological activities of RNAs $(58,63,64)$. The ESs of RNAs form by reshuffling base pairs in and around bulges and they tend to be enriched in helical residues, as unpaired nucleotides in bulges and internal loops from the dominant ground state (GS) form mismatches in the 
ES (Figure 1C). We also hypothesized that the degree to which an ES is stabilized relative to the GS could be controlled by changing the number of nucleotides that are modified. In this regard, it is interesting to note that many RNAs possess clusters of Nm-modified nucleotides $(2,53)$ that could have a synergistic effect on fine-tuning the conformational exchange.

By changing the abundance and possibly the lifetime of ESs, Nm modifications could change RNA ensembles without changing sequence, providing an additional chemical layer with which to optimize RNA structural dynamics for folding and function. In addition to potentially explaining some of the biological roles of $\mathrm{Nm}$, having an ability to control RNA conformational ensembles through $\mathrm{Nm}$ modifications may provide a valuable chemical tool for elucidating the characteristics of these lowabundance and short-lived conformations that are difficult to study (55). Here, we have tested our hypothesis using the well-characterized HIV-1 transactivation response element (TAR) RNA as a model system for non-canonical bulge motifs (65). Although TAR is not naturally modified with $\mathrm{Nm}$, its secondary structural ensemble in its unmodified form has been extensively characterized previously using NMR relaxation dispersion (RD) $(66,67)$. It therefore provides an excellent model system for testing our Nm-directed preferential stabilization of paired ESs hypothesis.

Prior NMR RD studies $(66,67)$ showed that TAR exists as a dynamic ensemble involving a GS and at least two ESs (ES1 and ES2) (Figure 1C) (68). ES1 forms rapidly $\left(k_{\mathrm{ex}}=k_{1}+k_{-1} \sim 25800 \mathrm{~s}^{-1}\right)$ by zipping up the apical loop to form two mismatches, and has a relatively high population ( $\mathrm{p}_{E S 1}$ ) of $\sim 13 \%$ (66). On the other hand, ES2 forms more slowly $\left(k_{\mathrm{ex}}=474 \mathrm{~s}^{-1}\right)$ through more extensive remodeling of the trinucleotide bulge, upper stem, and apical loop, and has a substantially lower population ( $\mathrm{p}_{\mathrm{ES} 2}$ $\sim 0.4 \%$ ) (67). Based on a recent NMR study (33), the unpaired bulge and apical loop residues C24, $\mathrm{U} 25$, and $\mathrm{A} 35$ are highly enriched in the $\mathrm{C} 2$ '-endo conformation in the GS whereas they are predominantly C3'-endo and helical in ES1 (A35) and ES2 (C24, U25, and A35). We reasoned that introducing $\mathrm{Nm}$ at these three nucleotides would stabilize both ESs relative to the GS. Additionally, we hypothesized that the extent of stabilization would be greater for ES2 than ES1, given that all three modifications favor ES2 whereas only Am35 favors ES1.

Indeed, NMR RD measurements showed that $\mathrm{Nm}$ increased both the abundance and the lifetime of TAR ES1 and ES2. Through a phi $(\Phi)$ value analysis (69-73), the impact of $\mathrm{Nm}$ on the exchange kinetics also allowed us to obtain rare conformational insights into the transition state (TS) for the GS-ES conformational exchange. Our results reveal a new mechanism by which $\mathrm{Nm}$ could alter 
the biological activity of Nm-modified RNAs as well as establish the utility of $\mathrm{Nm}$ as a chemical tool for the in-depth characterization of alternative ES RNA conformations.

\section{MATERIAL AND METHODS}

\section{Preparation of RNA samples}

Unlabeled TAR, TAR-A35 and TAR-C24U25A35 and ${ }^{15} \mathrm{~N}$ and ${ }^{13} \mathrm{C}$ site-labeled TAR and TARC24U25A35 oligonucleotides were synthesized using a MerMade 6 Oligo Synthesizer (BioAutomation) for solid-phase synthesis using standard phosphoramidite chemistry and deprotection protocols $(74,75)$. Unlabeled phosphoramidites were purchased from ChemGenes and site-labeled phosphoramidites were synthesized as described previously $(57,76)$. All RNA samples were prepared using 2'-TBDMS protected phosphoramidites using $1 \mu \mathrm{mol}$ standard synthesis columns (1000 $\AA$ ). The final 5'-DMT $\left(4,4^{\prime}-\right.$ dimethoxytrityl) was removed during the synthesis for DMT-off deprotection and PAGE purification. Removal of nucleobase protecting groups and cleavage from the $1 \mu \mathrm{mol}$ columns was achieved using $1 \mathrm{ml}$ of $30 \%$ ammonium hydroxide and $30 \%$ methylamine (1:1) followed by 2 hours incubation at room temperature. The solution was then air-dried. In order to remove the 2'-TBDMS protecting groups, 100 $\mu \mathrm{L}$ DMSO was added and the samples were heated at $65^{\circ} \mathrm{C}$ for $5 \mathrm{~min}$ to ensure the samples were fully dissolved. Then $125 \mu \mathrm{L}$ TEA-3HF was added and the samples were heated at $65^{\circ} \mathrm{C}$ for $2.5 \mathrm{~h}$. Samples were then precipitated overnight using $3 \mathrm{M}$ sodium acetate and $100 \%$ ethanol, air dried, then dissolved in water for gel purification. All RNA samples were purified using a $20 \%(\mathrm{w} / \mathrm{v})$ polyacrylamide gel with $8 \mathrm{M}$ urea and $1 \mathrm{X}$ Tris/borate/EDTA. RNA was removed from the excised gel by electro-elution in $1 \mathrm{x}$ Tris/acetic acid/EDTA followed by ethanol precipitation. The purified RNA was then annealed in water at a concentration of $50 \mu \mathrm{M}$ by heating at $95^{\circ} \mathrm{C}$ for $5 \mathrm{~min}$ followed by cooling on ice for $60 \mathrm{~min}$. The samples were then buffer exchanged five times using an Amicon Ultra-15 centrifugal filter (EMD Milipore) with a $3 \mathrm{kDa}$ cutoff into NMR buffer (15mM sodium phosphate, $25 \mathrm{mM} \mathrm{NaCl}, 0.1 \mathrm{mM}$ EDTA, $10 \%$ D2O) at $\mathrm{pH} 6.4$ with or without $1 / 3 \mathrm{mM} \mathrm{Mg}^{2+}$ for the site-labeled/unlabeled samples. The final concentration of unlabeled and site-labeled RNA NMR samples ranged between 0.7-2.0 mM and 0.3-0.7 mM, respectively. 


\section{UV optical melting experiments}

UV optical melting experiments were conducted on a PerkinElmer Lambda 25 UV/VIS spectrometer with RTP 6 Peltier Temperature Programmer and a PCB 1500 Water Peltier System. Samples with 3 $\mu M$ RNA were prepared by buffer exchanging the RNA into desired buffers (15mM sodium phosphate, $25 \mathrm{mM} \mathrm{NaCl}, 0.1 \mathrm{mM}$ EDTA, $\mathrm{pH} 6.4$ with or without $1 / 3 \mathrm{mM} \mathrm{Mg}^{2+}$ ) five times with a centrifugal concentrator (EMD Millipore). Optical melting experiments were performed in triplicates using a sample volume of $400 \mu \mathrm{L}$ in a Teflon-stoppered $1 \mathrm{~cm}$ path length quartz cell. The absorbance at $260 \mathrm{~nm}\left(\mathrm{~A}_{260}\right)$ was measured as a function of varying the temperature between 15 and $95{ }^{\circ} \mathrm{C}$ at a ramp of $1{ }^{\circ} \mathrm{C} / \mathrm{min}$. The absorbance curves were fit to two-state model to obtain the thermodynamic parameters using an inhouse Mathematica script and the following equations:

$A_{260}=\left(\left(\left(m_{d s} * T\right)+b_{d s}\right) * f_{T A R}\right)+\left(\left(\left(m_{s s} * T\right)+b_{s s}\right) *\left(1-f_{T A R}\right)\right)$

$f_{T A R}=\frac{e^{\left(\frac{1}{T_{m}}-\frac{1}{T}\right) \frac{\Delta H^{\circ}}{R}}}{1+e^{\left(\frac{1}{T_{m}}-\frac{1}{T}\right) \frac{\Delta H^{\circ}}{R}}}$

where $m_{d s}, b_{d s}, m_{s s}$ and $b_{s s}$ are coefficients representing the temperature dependence of the extinction coefficients of the folded and single-stranded species, $T$ is the temperature $(K), R$ is the gas constant $(\mathrm{kcal} / \mathrm{mol} / \mathrm{K}), \mathrm{f}_{\mathrm{TAR}}$ is the fraction of folded TAR at a given temperature and $\Delta \mathrm{H}^{\circ}$ is the enthalpy of annealing. The free energy and entropy of annealing, $\Delta G^{\circ}$ and $\Delta S^{\circ}$ were calculated using the following equation:

$\Delta S^{\circ}=\frac{\Delta H^{\circ}}{T_{m}} ; \Delta G^{\circ}=\Delta H^{\circ}-T \Delta S^{\circ}$

The uncertainty in $T_{\mathrm{m}}, \Delta \mathrm{H}^{\circ}, \Delta \mathrm{G}^{\circ}$, and $\Delta \mathrm{S}^{\circ}$ was obtained based on the standard deviation in triplicate measurements. Differences in the standard free energy, entropy, and enthalpy were computed as following:

$\Delta \Delta G^{\circ}=\Delta G^{\circ}($ Nm-modified $)-\Delta G^{\circ}$ (unmodified TAR)

$\Delta \Delta S^{\circ}=\Delta S^{\circ}($ Nm-modified $)-\Delta S^{\circ}$ (unmodified TAR)

$\Delta \Delta \mathrm{H}^{\circ}=\Delta \mathrm{H}^{\circ}(\mathrm{Nm}$-modified $)-\Delta \mathrm{H}^{\circ}$ (unmodified TAR $)$

For the triply modified Nm TAR samples with high ES populations, the lower baseline of the absorbance curves was seen to deviate from linearity. Interestingly, a statistically better fit to the data could be obtained using a three-state fit of the absorbance curves while considering the GS, ES and unfolded species as contributing to the measured absorbance (data not shown). Nevertheless, fitting including 
three-states was seen to minimally influence the stabilities relative to two-states fitting (data not shown). Details of this analysis are outside the scope of this study and will be discussed in future studies.

\section{NMR Experiments}

All NMR experiments were performed on a $600 \mathrm{MHz}$ Bruker NMR spectrometer equipped with an HCN cryogenic probe. Data were processed using NMRpipe (77) and analyzed using SPARKY (T.D. Goddard and D.G. Kneller, SPARKY 3, University of California, San Francisco). Resonances in Nmmodified TAR were assigned based on prior assignments of unmodified TAR (33) and confirmed using 2D HSQC, HMQC, and ${ }^{1} \mathrm{H}-{ }^{1} \mathrm{H}$ NOESY experiments (150 ms mixing time).

Determining ES1 population using chemical shift perturbation (CSP) analysis. Because the GS-ES1 exchange is fast on the NMR chemical shift timescale (66), the observed resonance $\left(\delta_{\text {obs }}\right)$ in the $2 D$ $\left[{ }^{13} \mathrm{C},{ }^{1} \mathrm{H}\right] \mathrm{HSQC}$ spectra of unmodified TAR corresponds to a population-weighted average between the chemical shifts of the GS $\left(\delta_{G S}\right)$ and ES1 $\left(\delta_{\mathrm{ES} 1}\right)$ :

$\delta_{\mathrm{obs}}=\left(1-\mathrm{p}_{\mathrm{ES} 1}\right) \delta_{\mathrm{GS}}+\mathrm{p}_{\mathrm{ES} 1} \delta_{\mathrm{ES} 1}$

in which 1-pES1 and $p_{E S 1}$ are the populations of the GS and ES1, respectively. For unmodified TAR, pES1 $\sim 13 \%$ and $\Delta \delta=\delta_{\mathrm{ES} 1}-\delta_{\mathrm{GS}}=2.6 \mathrm{ppm}$, as determined previously using ${ }^{13} \mathrm{C} R_{1 \rho} \mathrm{RD}(66)$. This allowed determination of $\delta_{\mathrm{GS}}$ and $\delta_{\mathrm{ES} 1}$ for the various spins that experience RD (C30-C1', U31-C1', U31-C6, G34-C1' and $\mathrm{G} 34-\mathrm{C} 8$ ). Note that $\mathrm{A} 35-\mathrm{C} 1{ }^{\prime}$ and $\mathrm{A} 35-\mathrm{C} 8$ are expected to exhibit CSPs towards ES1 chemical shifts; however, these resonances were excluded from analysis since they exhibit sizeable CSPs due to the chemical modification itself as verified using density functional theory (DFT) calculations (Table S1). Based on the $\delta_{\text {obs }}$ measured for $\mathrm{Nm}$-modified TAR, and the $\delta_{G S}$ and $\delta_{\text {ES1 }}$ values deduced for unmodified TAR, pES1 was computed for Nm-modified TAR for each of C30-C1', U31-C1', U31-C6, G34-C1' and G34-C8 (Table S2). Similar pES1 values (average $p_{E S 1}=28.7 \pm 1.5 \%$ in TAR-A35 and $28.8 \pm 3.1 \%$ in TAR-C24U25A35) were obtained for these spins in the Nm-modified TAR consistent with a concerted shift in population toward ES1. Here the uncertainty represents the standard deviation across the different spins. The same approach was used to estimate $p_{E S 1}$ for site-labeled G34-C8 TAR $\left(p_{E S 1}=7.3 \%\right)$ and TAR-C24U25A35 $\left(p_{E S 1}=14.5 \%\right)$ in $1 \mathrm{mM} \mathrm{Mg}^{2+}$ using the $\delta_{\mathrm{GS}}$ and $\delta_{\mathrm{ES} 1}$ values calculated for TAR in the absence of $\mathrm{Mg}^{2+}$ (Table S2). 
${ }^{13} \mathrm{C} /{ }^{15} \mathrm{~N} R_{1 \rho}$ experiments. ${ }^{13} \mathrm{C} /{ }^{15} \mathrm{~N} R_{1 \rho} \mathrm{RD}$ measurements were performed on site-labeled TAR and TARC24U25A35 RNA in the presence or absence of $1 \mathrm{mM} \mathrm{Mg}^{2+}$ as described previously (55). RD measurements on the unmodified chemically synthesized site-labeled TAR were used to confirm reproducibility with previously published data on uniformly ${ }^{13} \mathrm{C} /{ }^{15} \mathrm{~N}$ labeled TAR prepared using in vitro transcription $(66,67)$. The spin lock power and offset combinations used in $R_{1 \rho}$ experiments are listed in Table S7.

Analysis of ${ }^{13} \mathrm{C} /{ }^{15} \mathrm{~N} R_{1 \rho}$ data. The peak intensity in ${ }^{13} \mathrm{C} /{ }^{15} \mathrm{~N} R_{1 \rho}$ measurements at each relaxation delay was extracted using NMRPipe (77). The $R_{1 \rho}$ value was obtained by fitting the peak intensities at all delay points to a mono-exponential decay and the error was estimated by Monte-Carlo simulation with 500 iterations as described previously (55). The off-resonance $R_{1 \rho}$ data was fit to a two-state exchange model using the Bloch-McConnel equations and an in-house Python script with the fitting errors estimated by a Monte-Carlo scheme (55). The fitted exchange parameters included the population ( $\left.p_{B}\right)$, exchange rate $\left(k_{\text {ex }}\right)$, forward rate constant $\left(k_{1}\right)$, backward rate constant $\left(k_{-1}\right)$, and the difference in chemical shift between the ES and GS $\left(\Delta \omega=\omega_{\mathrm{ES}}-\omega_{\mathrm{GS}}\right)$. In all cases, the fitting assumed $R_{1, \mathrm{GS}}=R_{1, \mathrm{ES}}$ $=R_{1}$ and $R_{2, \mathrm{GS}}=R_{2, \mathrm{ES}}=R_{2}$. In the $R_{1 \rho}$ plots in Figures 3 and $4, \Omega \mathrm{OBS}=\omega \mathrm{OBS}-\omega \mathrm{RF}$, where $\omega \mathrm{OBS}$ is the Larmor frequency of the observed resonance and $\omega R F$ is angular frequency of the applied spin-lock power.

GS-ES1 exchange: For the fast exchanging ES1, both in absence and presence of $\mathrm{Mg}^{2+}$, the initial alignment of the magnetization was assumed to be along the average effective field of the GS and ES ( $\omega_{\text {eff,AVG }}$ ) when fitting data to the Bloch-McConnel equations (55). Because the GS-ES1 exchange is fast on the NMR chemical shift timescale (66), and only a single RD probe (G34-C8) was utilized, the exchange parameters, particularly $\mathrm{p}_{\mathrm{ES} 1}$, have high uncertainty (Table S3). Nevertheless, fitting the $R_{1 \rho}$ data measured for unmodified TAR in the absence of $\mathrm{Mg}^{2+}$ assuming the previously reported $\mathrm{p}_{\mathrm{ES} 1}(\sim$ 13\%) (66) yielded exchange parameters $\left(k_{\mathrm{ex}}, k_{1}, k_{-1}\right.$, and $\left.\Delta \omega\right)$ that are within error to those reported previously when using many RD probes and fully labeled TAR (Table S3). When fitting $R_{1 \rho}$ data for TAR in the presence of $1 \mathrm{mM} \mathrm{Mg}^{2+}$, the population was fixed to the value determined by CSP analysis (i.e. $7 \%$, Table S2). Because Nm slows down the GS-ES1 exchange, the exchange parameters for TAR-C24U25A35 have lower uncertainty (Table S3). Based on the two-state fit of the $R_{1 \rho}$ data, $\Delta \omega_{\mathrm{G} 34-}$ 
$\mathrm{c8}=2.4 \pm 0.2 \mathrm{ppm}$ for TAR-C24U25A35 in excellent agreement with values measured in unmodified $\operatorname{TAR}(2.6 \pm 0.2 \mathrm{ppm})$. The similar $\Delta \omega$ values indicates that the $\mathrm{Nm}$ does not lead to a new ES but rather changes the properties of the pre-existing ES1 (Table S3). The fit revealed that Nm increases the ES1 population $\sim 2$-fold, from $13 \%$ to $30 \%$ in the absence of $\mathrm{Mg}^{2+}$ and from $7 \%$ to $14 \%$ in the presence of $\mathrm{Mg}^{2+}$, in excellent agreement with the CSPs (Figure 3B and Table S3).

GS-ES2 exchange: Because GS-ES2 is in slow exchange, the initial magnetization was assumed to be aligned along the GS when fitting $R_{1 p}$ data to the Bloch-McConnel equations. Global fitting was carried by sharing $\mathrm{p}_{\mathrm{ES} 2}$ and $k_{\mathrm{ex}}$ for U23-C6 and U38-N3. Due to degeneracy arising from slow exchange (78), $R_{1 \rho}$ data measured for TAR in the absence of $\mathrm{Mg}^{2+}$ and for TAR-C24U25A35 in the absence and presence of $\mathrm{Mg}^{2+}$ was fit by fixing the population to the values determined using CEST (Table S6). Based on a two-state fit of the $R_{1 \rho}$ data, the $\Delta \omega$ values obtained for U23-C6 and U38-N3 were in very good agreement with values measured for unmodified TAR, indicating that the $\mathrm{Nm}$ does not lead to a new ES but rather changes the properties of the pre-existing ES2 (Table S6). The addition of $1 \mathrm{mM}$ $\mathrm{Mg}^{2+}$ does not significantly affect the chemical shifts of the ES2 probes (U23-C6 and U38-N3) used in this study (Figure S7). Therefore, the weak profiles measured for U23-C6 in the presence of $\mathrm{Mg}^{2+}$ (Figure 4B) can be attributed to a reduced $\Delta \omega$ due to a $\mathrm{Mg}^{2+}$ induced downfield shift in U23-C6 GS chemical shift (Figure S3D). A two-state fit of the U38-N3 data yielded $\Delta \omega$ values that are in very good agreement to those measured for TAR in the absence of $\mathrm{Mg}^{2+}$ confirming that $\mathrm{Mg}^{2+}$ does not lead to a new ES but rather changes the properties of the pre-existing ES2 (Table S6).

${ }^{13} \mathrm{C} /{ }^{15} \mathrm{~N}$ CEST experiment. All the ${ }^{13} \mathrm{C} /{ }^{15} \mathrm{~N}$ CEST measurements for probing GS-ES2 exchange were performed on site-specific labeled TAR (without $\mathrm{Mg}^{2+}$ ) and TAR-C24U25A35 RNA (with and without 1 $\mathrm{mM} \mathrm{Mg}^{2+}$ ) as described previously (78-80). The spin lock power and offset combinations used in CEST measurements are listed in Table S8. The relaxation delay was $0.2 \mathrm{sec}$ for U23-C6 (TAR, without $\mathrm{Mg}^{2+}$ ), U23-C6 (TAR-C24U25A35, without $\mathrm{Mg}^{2+}$ ), U38-N3 (TAR-C24U25A35, without $\mathrm{Mg}^{2+}$ ) and $0.3 \mathrm{sec}$ for U38-N3 (TAR, without $\mathrm{Mg}^{2+}$ ), U23-C6 (TAR-C24U25A35, with $\mathrm{Mg}^{2+}$ ), and U38-N3 (TAR-C24U25A35, with $\mathrm{Mg}^{2+}$ ). 
Analysis of ${ }^{13} \mathrm{C} /{ }^{15} \mathrm{~N}$ CEST data. For each spin lock power and offset, the peak intensities in the 1D ${ }^{13} \mathrm{C} /{ }^{15} \mathrm{~N}$ CEST were extracted using NMRPipe (77). The uncertainty in peak intensity was obtained from the intensity variations in the regions of CEST profiles that do not contain any intensity dips (80). The exchange parameters were obtained by fitting the data to the two-state Bloch-McConnel equations using an in-house Python script with the fitting errors estimated by a Monte-Carlo scheme with 100 iterations (78). In all cases, the observed peak was assumed to be the GS peak, as the system is in slow exchange regime (55). ES2 global fitting was carried out by sharing $\mathrm{p}_{\mathrm{B}}$ and $k_{\mathrm{ex}}$ between U23-C6 and U38-N3 and assuming $R_{1, \mathrm{GS}}=R_{1, \mathrm{ES}}=R_{1}$ and $R_{2, \mathrm{GS}}=R_{2, \mathrm{ES}}=R_{2}$. In the CEST profiles shown in Figure 4 and Figure S5, $\Omega=\omega_{\mathrm{RF}}-\omega_{\mathrm{OBS}}$ where $\omega_{\mathrm{OBS}}$ is the Larmor frequency of the observed resonance and $\omega_{R F}$ is angular frequency of the applied spin-lock power.

\section{Density functional theory calculations}

Density functional theory (DFT) calculations to compute chemical shifts were performed using Gaussian 09c (Gaussian Inc.) (81) as previously described (82). The starting structures of mononucleotide $\mathrm{dA} / \mathrm{rA}$ and $\mathrm{dT} / \mathrm{rU}$ were generated using the fiber module of the 3DNA suite (83). The structure of $\mathrm{rA} / \mathrm{rU}$ with C2'-endo sugar was derived from dA/dT by adding a 2'-hydroxl group on the deoxyribose and removing the 7-methyl group from dT using GaussView (https://gaussian.com/gaussview6). The structure of modified $\mathrm{rA} / \mathrm{rU}$ was generated by furtherly adding a 2'-O-methyl group on the ribose. In all cases, the phosphate backbone was truncated using GaussView, thus leaving only the nucleoside motifs with all heavy atoms fixed. Geometry optimization was performed using the B3LYP functional (84) with two runs using the $3-21 G(85)$ and $6-311+G(2 d, p)$ basis sets $(86) .{ }^{13} \mathrm{C}$ chemical shifts were then calculated using the GIAO method (87) using the B3LYP/6-311+G $(2 d, p)$ basis sets. The calculated chemical shifts were then referenced to isotropic magnetic shielding of TMS.

\section{Phi $(\Phi)$-value analysis}

$\Phi$-value analysis (70) was carried out by computing $\Phi$ (Table S5) using the following equation:

$\Phi=\left(\Delta \Delta G^{\circ}{ }_{T S-G S} / \Delta \Delta G^{\circ}{ }_{G S-E S}\right)$

$\Delta \Delta G^{\circ}{ }^{G S-E S}=\Delta G^{\circ}$ GS-ES, modified TAR $-\Delta G^{\circ}$ GS-ES, unmodified TAR

$\Delta \Delta \mathrm{G}^{\circ} \mathrm{TS}$-GS $=\Delta^{\ddagger} \mathrm{G}^{\circ} \mathrm{TS}$-GS, modified TAR $-\Delta^{\ddagger} \mathrm{G}^{\circ}$ TS-GS, unmodified TAR 
where $\Delta \Delta \mathrm{G}^{\circ} \mathrm{GS}$-ES is the change in the free-energy difference between GS and ES induced by the $\mathrm{Nm}$ modification obtained from ES population ( $\mathrm{p}_{\mathrm{ES}}$ ) obtained using $R_{1 \rho}$ and CEST experiments (Tables S3 and S6):

$$
\Delta G_{G S-E S}^{\circ}=-R T\left(\ln \left(\frac{k_{1} h}{k_{B} T}\right)-\ln \left(\frac{k_{-1} h}{k_{B} T}\right)\right)=-R T \ln \left(\frac{p_{E S}}{1-p_{E S}}\right)
$$

Where $\mathrm{R}$ is the gas constant, $\mathrm{T}$ is temperature, $h$ is Planck's constant, $k_{\mathrm{B}}$ is Boltzmann's constant, $k_{1}$ is the forward rate constant, and $k_{-1}$ backward rate constant.

$\Delta \Delta \mathrm{G}^{\circ} \mathrm{TS}$-GS is the change in the forward free-energy barrier $\left(\Delta^{\ddagger} \mathrm{G}^{\circ}\right)$ induced by $\mathrm{Nm}$ calculated using the forward rate constant $k_{1}$ obtained from $R_{1 \rho}$ and CEST experiments (Tables S3 and S6):

$\Delta^{\ddagger} G^{\circ}{ }_{T S-G S}=-R T \ln \left(\frac{k_{1} h}{\kappa k_{B} T}\right)$

where $\kappa$ is the transmission coefficient and is assumed to be unity. 


\section{RESULTS}

\section{$\mathrm{Nm}$ modifications have a small impact on the thermal stability of the TAR GS}

We first used UV melting experiments to assess the impact of the modification on the overall thermal stability of TAR, which is dominated by the GS (population $\sim 86 \%$ ) and to a lesser extent ES1 (population $\sim 13 \%$ ) (see Methods). Given that other post-transcriptional modifications such as $\mathrm{m}^{6} \mathrm{~A}$ have been shown to affect RNA thermal stability and structural dynamics in a $\mathrm{Mg}^{2+}$-dependent manner $(78,88,89)$, and that the TAR bulge binds $\mathrm{Mg}^{2+}$ in a unique conformation $(90,91)$, experiments were performed in the absence and presence of $\mathrm{Mg}^{2+}$.

Using solid-phase oligonucleotide synthesis, TAR samples were prepared in which A35 alone (TAR-A35) or in conjunction with C24 and U25 (TAR-C24U25A35) were Nm modified (Figure 1C). A third reference sample was also prepared lacking any modifications (TAR). The single Am35 modification had a negligible effect on the free energy of melting (Table 1 and Figure $2 \mathrm{~A}$ ) both in the absence $\left(\Delta \Delta \mathrm{G}^{\circ} \sim-0.1 \mathrm{kcal} / \mathrm{mol}\right)$ and presence $\left(\Delta \Delta \mathrm{G}^{\circ} \sim+0.2 \mathrm{kcal} / \mathrm{mol}\right)$ of $3 \mathrm{mM} \mathrm{Mg}^{2+}$. The triple modification slightly stabilized TAR by $\Delta \Delta \mathrm{G}^{\circ} \sim-0.3 \mathrm{kcal} / \mathrm{mol}$ in the absence of $\mathrm{Mg}^{2+}$ and resulted in an equally small degree of destabilization $\left(\Delta \Delta \mathrm{G}^{\circ} \sim+0.4 \mathrm{kcal} / \mathrm{mol}\right)$ in the presence of $3 \mathrm{mM} \mathrm{Mg}^{2+}$ (Table 1 and Figure 2A). The addition of $3 \mathrm{mM} \mathrm{Mg}^{2+}$ stabilized both $\mathrm{Nm}$-modified and unmodified TAR samples by a more significant amount of $\sim 3 \mathrm{kcal} / \mathrm{mol}$ (Table 1 ) consistent with prior studies $(90,91)$ showing that TAR interacts favorably with divalent metals.

These results show that even three $\mathrm{Nm}$ modifications have minor contributions to the overall thermal stability of RNAs when placed on unpaired flexible nucleotides. Such small energetic perturbations on the GS make the modification particularly attractive for carrying out $\Phi$-value analysis to gain insights into the transition-state (TS) (69-73). As we show below, while the modifications only weakly affected the energetic stability of the TAR folded state relative to the unfolded one, they had a bigger effect on the energetic stabilities of the two ESs relative to the GS, particularly for ES2.

\section{$\mathrm{Nm}$ modifications impact the TAR conformational preferences}

Comparison of the 1D ${ }^{1} \mathrm{H}$ NMR spectra of TAR-A35 and TAR-C24U25A35 with their unmodified counterparts (Figure 2B), revealed that $\mathrm{Nm}$ minimally impacts the secondary structure of the dominant GS both in the presence and absence of $3 \mathrm{mM} \mathrm{Mg}^{2+}$. Both $\mathrm{Nm}$-modified samples showed the imino resonances belonging to the TAR Watson-Crick base-pairs (bps) (Figure 2B). The modifications did 
however induce chemical shift perturbations (CSPs) in $1 \mathrm{D}{ }^{1} \mathrm{H}$ NMR (Figure $2 \mathrm{~B}$ ) and $2 \mathrm{D}\left[{ }^{13} \mathrm{C},{ }^{1} \mathrm{H}\right] \mathrm{HSQC}$ spectra (Figure 2C and Figures S1-S3), indicating that they do indeed affect the conformational dynamics of TAR. As described below, the CSPs observed for apical loop residues in the 2D $\left[{ }^{13} \mathrm{C},{ }^{1} \mathrm{H}\right]$ HSQC spectrum are as expected if the Nm modifications were to increase the abundance of ES1. Other sizeable CSPs observed at the sugar resonances of the Nm-modified residues (Figures S1-S3) can be attributed to the chemical modification itself, as verified using density functional theory (DFT) calculations (Table S1) (92). Small CSPs were also observed in TAR-C24U25A35 at residues in and around the bulge (grey residues in Figure $2 \mathrm{C}$ ), indicating a subtle change in the GS conformational ensemble.

\section{$\mathrm{Nm}$ increases the abundance of ES1 relative to the GS}

The GS-ES1 exchange is fast on the NMR chemical shift timescale. Consequently, if the Nm modification at A35 were to increase the abundance of ES1 without substantially slowing the exchange kinetics, we would expect to observe CSPs in apical loop residues that are directed specifically towards the ES $1{ }^{1} \mathrm{H}$ and ${ }^{13} \mathrm{C}$ chemical shifts, which have been determined previously (66). Furthermore, we would expect similar CSPs for both TAR-A35 and TAR-C24U25A35 given that only Am35 is expected to bias the equilibrium in favor of ES1 (Figure 1C). The magnitude of the shift should be proportional to the degree to which the modification increases the ES1 population (66).

Indeed, for both TAR-A35 and TAR-C24U25A35 in the absence of $\mathrm{Mg}^{2+},{ }^{1} \mathrm{H}$ and ${ }^{13} \mathrm{C}$ CSPs were observed at $\mathrm{C} 30-\mathrm{C} 11^{\prime}, \mathrm{U} 31-\mathrm{C} 11^{\prime}, \mathrm{U} 31-\mathrm{C} 6, \mathrm{G} 34-\mathrm{C} 1$ ' and $\mathrm{G} 34-\mathrm{C} 8$ that are specifically directed towards the ES1 chemical shifts (Figure 2C and Figure S2). As a negative control, no CSPs were observed at apical loop residues G32 and G33, which have similar chemical shifts in the GS and ES1 as they remain unpaired in the two cases (33) (Figure S2). Based on the magnitude of the CSPs (see Methods), Nm increases the ES1 population 2-fold from $13 \pm 2 \%$ to $29 \pm 3 \%$ in both TAR-A35 and TAR-C24U25A35 (Table S2). The $\sim 0.6 \mathrm{kcal} / \mathrm{mol}$ stabilization of ES1 relative to the GS (Figure S4) is larger than the $\sim 0.2$ $\mathrm{kcal} / \mathrm{mol}$ stabilization reported previously from incorporating a single Am into RNA duplexes (93). This is not surprising given that the net effect of $\mathrm{Nm}$ on thermodynamic parameters is context-dependent (93). The Am35 modification at the Am35+-C30 mismatch could have a more stabilizing effect on ES1 and/or the modification may also destabilize the unpaired conformation of A35 in the GS. 
To assess the ES1 abundance in the presence of $\mathrm{Mg}^{2+}$, we prepared unmodified TAR and TARC24U25A35 samples site-labeled $(57,76)$ with ${ }^{13} \mathrm{C}$ at G34-C8 to probe ES1, and with ${ }^{13} \mathrm{C}$ at U23-C6 and ${ }^{15} \mathrm{~N}$ at U38-N3 to probe ES2 (66) (see below). This was necessary to boost NMR sensitivity given that $\mathrm{Mg}^{2+}$ significantly broadened the resonances in the 2D NMR spectra (94) (Figure S3A-C). The addition of $1 \mathrm{mM} \mathrm{Mg}^{2+}$ to TAR resulted in CSPs at G34-C8H8 that are directed away from ES1 toward the GS, as expected if $\mathrm{Mg}^{2+}$ were to stabilize the GS relative to ES1 (Figure S3D). Based on the magnitude of these CSPs, $\mathrm{Mg}^{2+}$ reduced the abundance of ES1 two-fold (from 13\% to 7\%, Table S2) corresponding to $0.4 \mathrm{kcal} / \mathrm{mol}$ destabilization of ES1 relative to the GS (Figure S4). Thus, $\mathrm{Mg}^{2+}$ preferentially binds and stabilizes the apical loop in the more open GS conformation relative to the zipped up ES1.

Although $\mathrm{Mg}^{2+}$ decreased the ES1 population, introducing $\mathrm{Nm}$ once again induced CPSs directed towards ES1 (Figure S3D). Based on these CSPs, Nm increased the abundance of ES1 twofold (from $7 \%$ to $~ 14 \%$, Table S2). Therefore, Nm stabilizes ES1 relative to GS in a manner independent of $\mathrm{Mg}^{2+}$, whereas $\mathrm{Mg}^{2+}$ stabilizes the GS relative to ES1 in a manner independent of Nm (Figure S3D and Figure S4). This independence could arise because the $\mathrm{Mg}^{2+}$ ions that stabilize the GS relative to the ES1 interact with apical loop residues distant from the modified A35 site and are therefore unaffected by the $\mathrm{Nm}$ modification.

\section{$\mathrm{Nm}$ increases the lifetime of ES1 in the absence and presence of $\mathrm{Mg}^{2+}$}

To further confirm that $\mathrm{Nm}$ stabilizes ES1, and to gain insights into how $\mathrm{Nm}$ affects the GS-ES1 exchange kinetics, we preformed off-resonance spin relaxation in the rotating frame $\left(R_{1 \rho}\right)$ RD NMR experiments on the isotopically labeled unmodified TAR and modified TAR-C24U25A35 samples (Figure $3 \mathrm{~A}$ ). The $\mathrm{Nm}$ modification and $\mathrm{Mg}^{2+}$ had opposite effects on the $R_{1 \rho}$ profiles measured for $\mathrm{G} 34-$ C8. Nm enhanced the $R_{1 \rho}$ profile whereas the addition of $\mathrm{Mg}^{2+}$ diminished it (Figure 3A). In accord with the CSP analysis, a two-state fit of the RD data showed that Nm increased the ES1 population 2fold both in the presence and absence of $\mathrm{Mg}^{2+}$, whereas $\mathrm{Mg}^{2+}$ decreased the population by a similar amount in the presence and absence of Nm (Figure 3B and Table S3).

The RD data also revealed that $\mathrm{Nm}$ did not significantly change the forward rate (Figure 3C). Rather, it robustly reduced the backward rate $(k-1)$ (Figure 3D) and increased the ES1 lifetime by 2fold in the absence and presence of $\mathrm{Mg}^{2+}$ (Figure 3E). Conversely, $\mathrm{Mg}^{2+}$ robustly increased the 
abundance and lifetime of the GS in the absence and presence of $\mathrm{Nm}$ modifications. Thus, the opposing effects of $\mathrm{Nm}$ and $\mathrm{Mg}^{2+}$ on the GS-ES1 exchange are independent of one another at both the thermodynamic as well as the kinetic level. While this suggests that the modification similarly impacts the energetic stabilities of the GS and the transition state for ES1 (TS1) (Table S4 and Figure S4), the nature of the TS1 was difficult to ascertain given the large uncertainty in the $\Phi$-value (70) (Table S5).

\section{$\mathrm{Nm}$ increases the abundance and lifetime of ES2 in a $\mathrm{Mg}^{2+}$-dependent manner}

We next turned our attention to how Nm impacts the GS-ES2 exchange using U23-C6 and U38-N3 as RD probes. Here, we expected a greater effect because all three Nm modifications should energetically favor ES2 relative to the GS. To overcome a degeneracy arising due to slow exchange kinetics (Figure S5), we supplemented the $R_{1 \rho}$ data with measurements of chemical exchange saturation transfer (CEST) experiments $(79,80)$, which are better suited for studying slower GS-ES exchange process (78).

We first benchmarked the unmodified site-labeled TAR sample in the absence of $\mathrm{Mg}^{2+}$. As expected, the exchange parameters obtained by fitting CEST (Figure 4A) and $R_{1 \rho}$ (Figure 4B) data measured for U23-C6 and U38-N3 (Figure 4C-F and Table S6) were in excellent agreement with the values reported previously (67). As predicted, relative to unmodified TAR, TAR-C24U25A35 showed enhanced CEST (Figure 4A) and $R_{1 p}$ profiles (Figure 4B), consistent with an increase in ES2 population (Figure $4 \mathrm{C}$ ). A two-state fit of the $\mathrm{RD}$ data revealed that $\mathrm{Nm}$ increased the $\mathrm{ES} 2$ population $\sim 5$-fold (from $\sim 0.5 \%$ to $\sim 2.6 \%$ ), stabilizing ES2 relative to the GS by $\sim 1 \mathrm{kcal} / \mathrm{mol}$ (Table S6 and Figure S6). The $\sim 2.5-$ fold greater stabilization of ES2 relative to ES1 was as expected from application of three versus a single $\mathrm{Nm}$ modification. As with ES1, the Nm modifications increased ES2 lifetime by $\sim 2$-fold from $\sim 2.8$ to $\sim 6.0 \mathrm{~ms}$ (Table S6). However, unlike for ES1, the $\mathrm{Nm}$ modifications also increased the forward rate $\sim 2.3$-fold (Figure 4D and Table S6). This results in an intermediate $\Phi$-value of $\sim 0.5$ for Nm-modified TAR in the absence of $\mathrm{Mg}^{2+}$, which is difficult to interpret to obtain structural insights into the transition state for ES2 formation (TS2) (Table S5).

As with ES1, the addition of $\mathrm{Mg}^{2+}$ to unmodified TAR diminished the CEST and $R_{1 p}$ profiles of U38-N3 and U23-C6 (Figure 4B) due to a $\sim 4$-fold reduction in ES2 population (from $\sim 0.54$ to $\sim 0.14 \%$, Table S6). The greater degree to which $\mathrm{Mg}^{2+}$ stabilizes the GS relative to ES2 versus ES1 is as 
expected given that a change in the bulge conformation is required to form ES2 and that four divalent metal ions specifically bind and stabilize the TAR bulge in the GS conformation (91).

When combined together, $\mathrm{Nm}$ and $\mathrm{Mg}^{2+}$ produced an unexpected result. Based on the $R_{1 \rho}$ and CEST profiles, introducing the $\mathrm{Nm}$ modifications in the presence of $\mathrm{Mg}^{2+}$ increased the abundance of ES2 by 10 -fold (Figure 4C, 4F and Table S6). The greater stabilization of ES2 relative to the GS in the presence $\left(\Delta \Delta G^{\circ}=1.4 \mathrm{kcal} / \mathrm{mol}\right)$ versus absence of $\mathrm{Mg}^{2+}\left(\Delta \Delta \mathrm{G}^{\circ}=1.0 \mathrm{kcal} / \mathrm{mol}\right)$ might in part be explained by the UV melting data (Figure $2 \mathrm{~A}$ and Table 1), where Nm slightly destabilizes TAR GS in the presence of $\mathrm{Mg}^{2+}$ by $\sim 0.4 \mathrm{kcal} / \mathrm{mol}$. Consequently, the $\mathrm{Nm}$ modifications at the bulge could potentially be disrupting favorable $\mathrm{Mg}^{2+}$ interactions, thus destabilizing the $\mathrm{GS}$ and stabilizing the helical conformations formed in the ES2. This underscores the importance of examining how the $\mathrm{Nm}$ modifications may impact the conformational properties of non-canonical motifs.

Strikingly, in the presence of $\mathrm{Mg}^{2+}$, the introduction of the three $\mathrm{Nm}$ sites minimally impacted the forward rate $(1.2$-fold), whereas they decreased the backward rate by 12 -fold and increased the ES2 lifetime by the same amount. Compared to the unmodified TAR ES2 in the presence of $\mathrm{Mg}^{2+}$, the addition of $\mathrm{Nm}$ leads to a $\Phi$-value of $-0.07 \pm 0.10$ (Table S5) that strongly implies an early transition state for ES2 formation. These results indicate that in the presence of $\mathrm{Mg}^{2+}$, the $\mathrm{Nm}$ modified bulge residues C24 and U25 remain unpaired and enriched in C2'-endo sugar pucker in the TS2 as is observed in the GS.

\section{Direct observation of ES2 in Nm-modified TAR}

If three $\mathrm{Nm}$ modifications do indeed increase the population of ES2 to $>1 \%$, it should be possible to directly observe ES2 in the NMR spectra of TAR-C24U25A35, given that the GS-ES2 exchange is slow on the NMR chemical shift timescale, and is further slowed down by the $\mathrm{Nm}$ modifications. Indeed, a minor imino resonance was observed in $2 \mathrm{D}\left[{ }^{15} \mathrm{~N},{ }^{1} \mathrm{H}\right] \mathrm{HSQC}$ spectra of the sitelabeled TAR-C24U25A35 but not in unmodified TAR that could be assigned to U38 when forming the wobble U38-U25 mismatch in ES2 both in the absence (Figure 5A) and presence of $1 \mathrm{mM} \mathrm{Mg}^{2+}$ (Figure 5B). This resonance shows excellent overlap in both ${ }^{1} \mathrm{H}$ and ${ }^{15} \mathrm{~N}$ chemical shifts with the imino resonance for U38 in a previously described unmodified ES2-mutant (UUCG-ES2) (67) which stabilizes ES2 as the major conformation (Figure S7). Based on integrated volumes of the GS and ES2 resonances, the ES2 population is estimated to be $\sim 3 \%$ and $\sim 1 \%$ in the absence and presence of $\mathrm{Mg}^{2+}$, 
respectively, in excellent agreement with the ES2 populations ( $3 \%$ and $\sim 1 \%$, respectively) obtained from the CEST and $R_{1 \rho}$ data. Thus, Nm increases the abundance of ES2 to a degree that makes it directly observable in NMR 2D spectra.

\section{Discussion}

Our results show that beyond biasing the local sugar pucker equilibrium, $\mathrm{Nm}$ can have broader effects on RNA secondary structural ensembles by preferentially stabilizing and prolonging the lifetime of conformations where Nm-modified residues adopt a paired helical conformation. Interestingly, in the U2-U6 snRNA complex, Nm modifications stabilize a four-way junction where the $\mathrm{Nm}$-modified residues are paired relative to a three-way junction where they are unpaired (19), suggesting a similar mechanism to the one uncovered in our studies. Given the prevalence of secondary structural transitions during the folding, assembly, and function of both the spliceosome (95) and ribosome (96), and given the high abundance of $\mathrm{Nm}$ at functionally important non-canonical motifs, there may be analogous roles for $\mathrm{Nm}$ that involve the modulation of secondary structural equilibria in these and other Nm-modified RNAs. The preferential stabilization of paired conformations uncovered in this work should provide a useful framework for investigating such roles for Nm in RNA biology.

$\mathrm{Nm}$ also expands the toolbox of modifications currently available for studying RNA ESs (55). Point substitution mutations and nucleobase modifications that render an ES the dominant conformation have proven critically important for visualizing the structures of RNA ESs as well as for characterizing their functional properties $(55,66,67,97-99)$ and testing their validity as drug targets $(64)$. Yet because the sequence is altered, ES-mutants may not fully recapitulate the properties of the wild-type ES and finding an ES-mutant is not always feasible $(58,99)$. Any putative model for an RNA ES could be tested by introducing $\mathrm{Nm}$ at residues that are unpaired in the GS but are proposed to be paired in the ES and then testing for an increase in ES abundance by NMR or chemical probing (100-102). Nm is an attractive strategy for rationally modulating RNA ensembles because it preserves the sequence identify, can be used to target all four nucleotides, and because the modification is inexpensive and commercially available for in vitro studies. In addition, snoRNAs can be engineered to guide novel $\mathrm{Nm}$ modifications in vivo (103), and this could provide a route for studying RNA ESs in vivo or provide a means for altering RNA cellular activity as a therapeutic strategy. 
$\mathrm{Nm}$ also provides a rare opportunity to gain insights into the TS of conformational exchange through $\Phi$-value analysis, and specifically, to gain insights into whether or not a given residue is paired (C3'-endo) or unpaired (enriched in C2'-endo) in the TS. The results for ES2 in the presence of $\mathrm{Mg}^{2+}$ suggest an early TS2 in which all the modified residues remain unpaired and enriched in the C2'-endo sugar pucker as in the GS. An Nm scan across the TAR sequence combined with RD measurements will permit broader characterization of the TS structure, and help to elucidate the base pairs that might partially melt in the TS to initiate pair reshuffling and produce ES2. These structural insights into the TS2 are of great interest in the development of anti-HIV therapeutics given that the stabilization of ES2 potently inhibits the cellular activity of TAR (97) and that stabilizing the TS2 in addition to ES2 by small molecules would provide a route for accelerating production of the inhibitory ES2 conformation.

As is the case for other post-transcriptional modifications such as $\mathrm{m}^{6} \mathrm{~A}(78,88,89)$, the impact of $\mathrm{Nm}$ on the TAR dynamic ensemble cannot be fully understood without an appreciation for how $\mathrm{Mg}^{2+}$ impacts the ensemble as well (Figure 6). Whereas Nm perturbed the GS-ES1 exchange in a largely $\mathrm{Mg}^{2+}$ independent manner, both the kinetics and thermodynamics of GS-ES2 exchange were strongly dependent on $\mathrm{Mg}^{2+}$. Based on the crystal structure of TAR bound to $\mathrm{Ca}^{2+}(91)$, there are no direct contacts between bound metal ions and the 2'-OH of the Nm-modified residues (91). Rather, Nm modifications could potentially destabilize conformations within the TAR GS ensemble that preferentially bind $\mathrm{Mg}^{2+}$. Further studies are needed to understand how this inter-play between $\mathrm{Nm}$ and $\mathrm{Mg}^{2+}$ impacts RNA folding and function.

\section{SUPPLEMENTARY DATA}

Supplementary Data are available at NAR online.

\section{ACKNOWLEDGEMENT}

We thank the Duke Magnetic Resonance Spectroscopy Center for their technical support and resources. We also thank Atul K. Rangadurai and Megan Kelly for technical assistance and support and Dr. Richard Brennan for providing access to the UV-Vis spectrophotometer.

\section{FUNDING}

This work was supported by US National Institutes of Health [1R01GM132899 and U54GM103297 to H.M.A, T32HL007101 to H.A.A.] Duke Strong Start Award to C.L.H, and the Austrian Science Fund [P30370 and P32773 to C.K]. 


\section{CONFLICT OF INTEREST}

The authors declare the following competing financial interest(s): H.M.A. is an advisor to and holds an ownership interest in Nymirum, an RNA-based drug discovery company. The remaining authors declare no competing interests.

\section{REFERENCES}

1. Marchand, V., Pichot, F., Thuring, K., Ayadi, L., Freund, I., Dalpke, A., Helm, M. and Motorin, Y. (2017) Next-Generation Sequencing-Based RiboMethSeq Protocol for Analysis of tRNA 2'O-Methylation. Biomolecules, 7.

2. Taoka, M., Nobe, Y., Yamaki, Y., Sato, K., Ishikawa, H., Izumikawa, K., Yamauchi, Y., Hirota, K., Nakayama, H., Takahashi, N. et al. (2018) Landscape of the complete RNA chemical modifications in the human 80 S ribosome. Nucleic Acids Res, 46, 9289-9298.

3. Krogh, N., Kongsbak-Wismann, M., Geisler, C. and Nielsen, H. (2017) Substoichiometric ribose methylations in spliceosomal snRNAs. Org Biomol Chem, 15, 8872-8876.

4. Dai, Q., Moshitch-Moshkovitz, S., Han, D., Kol, N., Amariglio, N., Rechavi, G., Dominissini, D. and $\mathrm{He}, \mathrm{C}$. (2017) Nm-seq maps 2'-O-methylation sites in human mRNA with base precision. Nat Methods, 14, 695-698.

5. Elliott, B.A., Ho, H.T., Ranganathan, S.V., Vangaveti, S., llkayeva, O., Abou Assi, H., Choi, A.K., Agris, P.F. and Holley, C.L. (2019) Modification of messenger RNA by 2'-O-methylation regulates gene expression in vivo. Nat Commun, 10, 3401.

6. Somme, J., Van Laer, B., Roovers, M., Steyaert, J., Versees, W. and Droogmans, L. (2014) Characterization of two homologous 2'-O-methyltransferases showing different specificities for their tRNA substrates. RNA, 20, 1257-1271.

7. Cavaille, J., Nicoloso, M. and Bachellerie, J.P. (1996) Targeted ribose methylation of RNA in vivo directed by tailored antisense RNA guides. Nature, 383, 732-735.

8. Kiss-Laszlo, Z., Henry, Y., Bachellerie, J.P., Caizergues-Ferrer, M. and Kiss, T. (1996) Sitespecific ribose methylation of preribosomal RNA: a novel function for small nucleolar RNAs. Cell, 85, 1077-1088.

9. Dimitrova, D.G., Teysset, L. and Carre, C. (2019) RNA 2'-O-Methylation (Nm) Modification in Human Diseases. Genes (Basel), 10.

10. Ayadi, L., Galvanin, A., Pichot, F., Marchand, V. and Motorin, Y. (2019) RNA ribose methylation (2'-O-methylation): Occurrence, biosynthesis and biological functions. Biochim Biophys Acta Gene Regul Mech, 1862, 253-269.

11. Koh, C.M., Gurel, B., Sutcliffe, S., Aryee, M.J., Schultz, D., Iwata, T., Uemura, M., Zeller, K.I., Anele, U., Zheng, Q. et al. (2011) Alterations in nucleolar structure and gene expression programs in prostatic neoplasia are driven by the MYC oncogene. Am J Pathol, 178, 18241834.

12. Marcel, V., Ghayad, S.E., Belin, S., Therizols, G., Morel, A.P., Solano-Gonzalez, E., Vendrell, J.A., Hacot, S., Mertani, H.C., Albaret, M.A. et al. (2013) p53 acts as a safeguard of translational control by regulating fibrillarin and rRNA methylation in cancer. Cancer Cell, 24, 318-330.

13. Nagasawa, C., Ogren, A., Kibiryeva, N., Marshall, J., O'Brien, J.E., Kenmochi, N. and Bittel, D.C. (2018) The Role of scaRNAs in Adjusting Alternative mRNA Splicing in Heart Development. J Cardiovasc Dev Dis, 5.

14. Guy, M.P., Shaw, M., Weiner, C.L., Hobson, L., Stark, Z., Rose, K., Kalscheuer, V.M., Gecz, J. and Phizicky, E.M. (2015) Defects in tRNA Anticodon Loop 2'-O-Methylation Are Implicated 
in Nonsyndromic X-Linked Intellectual Disability due to Mutations in FTSJ1. Hum Mutat, 36, 1176-1187.

15. Piekna-Przybylska, D., Decatur, W.A. and Fournier, M.J. (2008) The 3D rRNA modification maps database: with interactive tools for ribosome analysis. Nucleic Acids Res, 36, D178-183.

16. Monaco, P.L., Marcel, V., Diaz, J.J. and Catez, F. (2018) 2'-O-Methylation of Ribosomal RNA: Towards an Epitranscriptomic Control of Translation? Biomolecules, 8.

17. Erales, J., Marchand, V., Panthu, B., Gillot, S., Belin, S., Ghayad, S.E., Garcia, M., Laforets, F., Marcel, V., Baudin-Baillieu, A. et al. (2017) Evidence for rRNA 2'-O-methylation plasticity: Control of intrinsic translational capabilities of human ribosomes. Proc Natl Acad Sci, 114, 12934-12939.

18. Donmez, G., Hartmuth, K. and Luhrmann, R. (2004) Modified nucleotides at the 5 ' end of human U2 snRNA are required for spliceosomal E-complex formation. RNA, 10, 1925-1933.

19. Karunatilaka, K.S. and Rueda, D. (2014) Post-transcriptional modifications modulate conformational dynamics in human U2-U6 snRNA complex. RNA, 20, 16-23.

20. Wang, X., Li, Z.T., Yan, Y., Lin, P., Tang, W., Hasler, D., Meduri, R., Li, Y., Hua, M.M., Qi, H.T. et al. (2020) LARP7-Mediated U6 snRNA Modification Ensures Splicing Fidelity and Spermatogenesis in Mice. Mol Cell, 77, 999-1013 e1016.

21. Hasler, D., Meduri, R., Bak, M., Lehmann, G., Heizinger, L., Wang, X., Li, Z.T., Sement, F.M., Bruckmann, A., Dock-Bregeon, A.C. et al. (2020) The Alazami Syndrome-Associated Protein LARP7 Guides U6 Small Nuclear RNA Modification and Contributes to Splicing Robustness. Mol Cell, 77, 1014-1031 e1013.

22. O'Brien, J.E., Jr., Kibiryeva, N., Zhou, X.G., Marshall, J.A., Lofland, G.K., Artman, M., Chen, J. and Bittel, D.C. (2012) Noncoding RNA expression in myocardium from infants with tetralogy of Fallot. Circ Cardiovasc Genet, 5, 279-286.

23. Patil, P., Kibiryeva, N., Uechi, T., Marshall, J., O'Brien, J.E., Jr., Artman, M., Kenmochi, N. and Bittel, D.C. (2015) scaRNAs regulate splicing and vertebrate heart development. Biochim Biophys Acta, 1852, 1619-1629.

24. Roost, C., Lynch, S.R., Batista, P.J., Qu, K., Chang, H.Y. and Kool, E.T. (2015) Structure and thermodynamics of N6-methyladenosine in RNA: a spring-loaded base modification. J Am Chem Soc, 137, 2107-2115.

25. Desrosiers, R., Friderici, K. and Rottman, F. (1974) Identification of methylated nucleosides in messenger RNA from Novikoff hepatoma cells. Proc Natl Acad Sci, 71, 3971-3975.

26. Rottman, F., Shatkin, A.J. and Perry, R.P. (1974) Sequences containing methylated nucleotides at the $5^{\prime}$ termini of messenger RNAs: possible implications for processing. Cell, $\mathbf{3}$, 197-199.

27. Meyer, K.D., Saletore, Y., Zumbo, P., Elemento, O., Mason, C.E. and Jaffrey, S.R. (2012) Comprehensive analysis of mRNA methylation reveals enrichment in 3' UTRs and near stop codons. Cell, 149, 1635-1646.

28. Meyer, K.D. and Jaffrey, S.R. (2017) Rethinking m(6)A Readers, Writers, and Erasers. Annu Rev Cell Dev Biol, 33, 319-342.

29. Dominissini, D., Moshitch-Moshkovitz, S., Schwartz, S., Salmon-Divon, M., Ungar, L., Osenberg, S., Cesarkas, K., Jacob-Hirsch, J., Amariglio, N., Kupiec, M. et al. (2012) Topology of the human and mouse m6A RNA methylomes revealed by m6A-seq. Nature, 485, 201-206.

30. Roundtree, I.A., Luo, G.Z., Zhang, Z., Wang, X., Zhou, T., Cui, Y., Sha, J., Huang, X., Guerrero, L., Xie, P. et al. (2017) YTHDC1 mediates nuclear export of N(6)-methyladenosine methylated mRNAs. Elife, 6.

31. Shi, H., Wang, X., Lu, Z., Zhao, B.S., Ma, H., Hsu, P.J., Liu, C. and He, C. (2017) YTHDF3 facilitates translation and decay of N(6)-methyladenosine-modified RNA. Cell Res, 27, 315328.

32. Davies, D.B. (1978) Conformations of nucleosides and nucleotides. Prog. Nucl. Magn. Reson. Spectrosc., 12, 135-225. 
33. Clay, M.C., Ganser, L.R., Merriman, D.K. and Al-Hashimi, H.M. (2017) Resolving sugar puckers in RNA excited states exposes slow modes of repuckering dynamics. Nucleic Acids Res, 45, e134.

34. Kawai, G., Yamamoto, Y., Kamimura, T., Masegi, T., Sekine, M., Hata, T., limori, T., Watanabe, T., Miyazawa, T. and Yokoyama, S. (1992) Conformational rigidity of specific pyrimidine residues in tRNA arises from posttranscriptional modifications that enhance steric interaction between the base and the 2'-hydroxyl group. Biochemistry, 31, 1040-1046.

35. Malek-Adamian, E., Patrascu, M.B., Jana, S.K., Martinez-Montero, S., Moitessier, N. and Damha, M.J. (2018) Adjusting the Structure of 2'-Modified Nucleosides and Oligonucleotides via C4'-alpha-F or C4'-alpha-OMe Substitution: Synthesis and Conformational Analysis. J Org Chem, 83, 9839-9849.

36. Cheng, D.M. and Sarma, R.H. (1977) Nuclear magnetic resonance study of the impact of ribose 2'-O-methylation on the aqueous solution conformation of cytidylyl-(3' leads to 5')cytidine. Biopolymers, 16, 1687-1711.

37. Mahto, S.K. and Chow, C.S. (2008) Synthesis and solution conformation studies of the modified nucleoside $\mathrm{N}(4), 2^{\prime}-$-O-dimethylcytidine $(\mathrm{m}(4) \mathrm{Cm})$ and its analogues. Bioorg Med Chem, 16, 8795-8800.

38. Yildirim, I., Kierzek, E., Kierzek, R. and Schatz, G.C. (2014) Interplay of LNA and 2'-O-methyl RNA in the structure and thermodynamics of RNA hybrid systems: a molecular dynamics study using the revised AMBER force field and comparison with experimental results. $J$ Phys Chem $B, 118,14177-14187$.

39. Kool, E.T. (1997) Preorganization of DNA: Design Principles for Improving Nucleic Acid Recognition by Synthetic Oligonucleotides. Chem Rev, 97, 1473-1488.

40. Auffinger, P. and Westhof, E. (2001) Hydrophobic Groups Stabilize the Hydration Shell of 2'O-Methylated RNA Duplexes. Angewandte Chemie, 40, 4648-4650.

41. Khvorova, A. and Watts, J.K. (2017) The chemical evolution of oligonucleotide therapies of clinical utility. Nat Biotechnol, 35, 238-248.

42. Ng, E.W., Shima, D.T., Calias, P., Cunningham, E.T., Jr., Guyer, D.R. and Adamis, A.P. (2006) Pegaptanib, a targeted anti-VEGF aptamer for ocular vascular disease. Nat Rev Drug Discov, 5, 123-132.

43. Shen, X. and Corey, D.R. (2018) Chemistry, mechanism and clinical status of antisense oligonucleotides and duplex RNAs. Nucleic Acids Res, 46, 1584-1600.

44. Khvorova, A. (2017) Oligonucleotide Therapeutics - A New Class of Cholesterol-Lowering Drugs. N Engl J Med, 376, 4-7.

45. Allerson, C.R., Sioufi, N., Jarres, R., Prakash, T.P., Naik, N., Berdeja, A., Wanders, L., Griffey, R.H., Swayze, E.E. and Bhat, B. (2005) Fully 2'-modified oligonucleotide duplexes with improved in vitro potency and stability compared to unmodified small interfering RNA. J Med Chem, 48, 901-904.

46. Jackson, A.L., Burchard, J., Leake, D., Reynolds, A., Schelter, J., Guo, J., Johnson, J.M., Lim, L., Karpilow, J., Nichols, K. et al. (2006) Position-specific chemical modification of siRNAs reduces "off-target" transcript silencing. RNA, 12, 1197-1205.

47. Lennox, K.A. and Behlke, M.A. (2011) Chemical modification and design of anti-miRNA oligonucleotides. Gene Ther, 18, 1111-1120.

48. Polikanov, Y.S., Melnikov, S.V., Soll, D. and Steitz, T.A. (2015) Structural insights into the role of rRNA modifications in protein synthesis and ribosome assembly. Nat Struct Mol Biol, 22, 342-344.

49. Didychuk, A.L., Butcher, S.E. and Brow, D.A. (2018) The life of U6 small nuclear RNA, from cradle to grave. RNA, 24, 437-460.

50. Montemayor, E.J., Curran, E.C., Liao, H.H., Andrews, K.L., Treba, C.N., Butcher, S.E. and Brow, D.A. (2014) Core structure of the U6 small nuclear ribonucleoprotein at 1.7-A resolution. Nat Struct Mol Biol, 21, 544-551. 
51. Mefford, M.A. and Staley, J.P. (2009) Evidence that U2/U6 helix I promotes both catalytic steps of pre-mRNA splicing and rearranges in between these steps. RNA, 15, 1386-1397.

52. Choi, J., Indrisiunaite, G., DeMirci, H., leong, K.W., Wang, J., Petrov, A., Prabhakar, A., Rechavi, G., Dominissini, D., He, C. et al. (2018) 2'-O-methylation in mRNA disrupts tRNA decoding during translation elongation. Nat Struct Mol Biol, 25, 208-216.

53. Bohnsack, M.T. and Sloan, K.E. (2018) Modifications in small nuclear RNAs and their roles in spliceosome assembly and function. Biol Chem, 399, 1265-1276.

54. Incarnato, D., Anselmi, F., Morandi, E., Neri, F., Maldotti, M., Rapelli, S., Parlato, C., Basile, G. and Oliviero, S. (2017) High-throughput single-base resolution mapping of RNA 2-Omethylated residues. Nucleic Acids Res, 45, 1433-1441.

55. Rangadurai, A., Szymaski, E.S., Kimsey, I.J., Shi, H. and Al-Hashimi, H.M. (2019) Characterizing micro-to-millisecond chemical exchange in nucleic acids using off-resonance R1p relaxation dispersion. Prog. Nucl. Magn. Reson. Spectrosc., 112-113, 55-102.

56. Zhao, B. and Zhang, Q. (2015) Characterizing excited conformational states of RNA by NMR spectroscopy. Curr Opin Struct Biol, 30, 134-146.

57. Juen, M.A., Wunderlich, C.H., Nussbaumer, F., Tollinger, M., Kontaxis, G., Konrat, R., Hansen, D.F. and Kreutz, C. (2016) Excited States of Nucleic Acids Probed by Proton Relaxation Dispersion NMR Spectroscopy. Angewandte Chemie, 55, 12008-12012.

58. Zhao, B., Guffy, S.L., Williams, B. and Zhang, Q. (2017) An excited state underlies gene regulation of a transcriptional riboswitch. Nat Chem Biol, 13, 968-974.

59. Chen, B., LeBlanc, R. and Dayie, T.K. (2016) SAM-II Riboswitch Samples at least Two Conformations in Solution in the Absence of Ligand: Implications for Recognition. Angewandte Chemie, 55, 2724-2727.

60. Rinnenthal, J., Buck, J., Ferner, J., Wacker, A., Furtig, B. and Schwalbe, H. (2011) Mapping the landscape of RNA dynamics with NMR spectroscopy. Acc Chem Res, 44, 1292-1301.

61. Sekhar, A. and Kay, L.E. (2013) NMR paves the way for atomic level descriptions of sparsely populated, transiently formed biomolecular conformers. Proc Natl Acad Sci, 110, 1286712874.

62. Tian, S., Cordero, P., Kladwang, W. and Das, R. (2014) High-throughput mutate-map-rescue evaluates SHAPE-directed RNA structure and uncovers excited states. RNA, 20, 1815-1826.

63. Kimsey, I.J., Petzold, K., Sathyamoorthy, B., Stein, Z.W. and Al-Hashimi, H.M. (2015) Visualizing transient Watson-Crick-like mispairs in DNA and RNA duplexes. Nature, 519, 315320.

64. Ganser, L.R., Kelly, M.L., Herschlag, D. and Al-Hashimi, H.M. (2019) The roles of structural dynamics in the cellular functions of RNAs. Nat Rev Mol Cell Biol, 20, 474-489.

65. Puglisi, J.D., Tan, R., Calnan, B.J., Frankel, A.D. and Williamson, J.R. (1992) Conformation of the TAR RNA-arginine complex by NMR spectroscopy. Science, 257, 76-80.

66. Dethoff, E.A., Petzold, K., Chugh, J., Casiano-Negroni, A. and Al-Hashimi, H.M. (2012) Visualizing transient low-populated structures of RNA. Nature, 491, 724-728.

67. Lee, J., Dethoff, E.A. and Al-Hashimi, H.M. (2014) Invisible RNA state dynamically couples distant motifs. Proc Natl Acad Sci, 111, 9485-9490.

68. Mulder, F.A., Mittermaier, A., Hon, B., Dahlquist, F.W. and Kay, L.E. (2001) Studying excited states of proteins by NMR spectroscopy. Nat Struct Biol, 8, 932-935.

69. Alvey, H.S., Gottardo, F.L., Nikolova, E.N. and Al-Hashimi, H.M. (2014) Widespread transient Hoogsteen base pairs in canonical duplex DNA with variable energetics. Nat Commun, 5, 4786.

70. Fersht, A.R., Matouschek, A. and Serrano, L. (1992) The folding of an enzyme. I. Theory of protein engineering analysis of stability and pathway of protein folding. $J \mathrm{Mol}$ Biol, 224, 771782. 
71. Neudecker, P., Zarrine-Afsar, A., Davidson, A.R. and Kay, L.E. (2007) Phi-value analysis of a three-state protein folding pathway by NMR relaxation dispersion spectroscopy. Proc NatI Acad Sci, 104, 15717-15722.

72. Bartley, L.E., Zhuang, X., Das, R., Chu, S. and Herschlag, D. (2003) Exploration of the transition state for tertiary structure formation between an RNA helix and a large structured RNA. J Mol Biol, 328, 1011-1026.

73. Cho, J.H., O'Connell, N., Raleigh, D.P. and Palmer, A.G., 3rd. (2010) Phi-value analysis for ultrafast folding proteins by NMR relaxation dispersion. J Am Chem Soc, 132, 450-451.

74. Damha, M.J. and Ogilvie, K.K. (1993) Oligoribonucleotide synthesis. The silylphosphoramidite method. Methods Mol Biol, 20, 81-114.

75. Ogilvie, K.K., Beaucage, S.L., Schifman, A.L., Theriault, N.Y. and Sadana, K.L. (1978) Synthesis of Oligoribonucleotides .2. Use of Silyl Protecting Groups in Nucleoside and Nucleotide Chemistry .7. Can J Chem, 56, 2768-2780.

76. Wunderlich, C.H., Spitzer, R., Santner, T., Fauster, K., Tollinger, M. and Kreutz, C. (2012) Synthesis of (6-(13)C)pyrimidine nucleotides as spin-labels for RNA dynamics. J Am Chem Soc, 134, 7558-7569.

77. Delaglio, F., Grzesiek, S., Vuister, G.W., Zhu, G., Pfeifer, J. and Bax, A. (1995) Nmrpipe - a Multidimensional Spectral Processing System Based on Unix Pipes. J. Biomol. NMR, 6, 277293.

78. Shi, H., Liu, B., Nussbaumer, F., Rangadurai, A., Kreutz, C. and Al-Hashimi, H.M. (2019) NMR Chemical Exchange Measurements Reveal That N(6)-Methyladenosine Slows RNA Annealing. J Am Chem Soc, 141, 19988-19993.

79. Zhao, B., Hansen, A.L. and Zhang, Q. (2014) Characterizing slow chemical exchange in nucleic acids by carbon CEST and low spin-lock field R(1rho) NMR spectroscopy. J Am Chem Soc, 136, 20-23.

80. Vallurupalli, P., Bouvignies, G. and Kay, L.E. (2012) Studying "invisible" excited protein states in slow exchange with a major state conformation. J. Am. Chem. Soc., 134, 8148-8161.

81. M. J. Frisch, G. W. Trucks, H. B. Schlegel, G. E. Scuseria, M. A. Robb, J. R. Cheeseman, G. Scalmani, V. Barone, G. A. Petersson, H. Nakatsuji et al. (2016) Gaussian 09, Revision D.01. Gaussian Inc, Wallingford CT.

82. Rangadurai, A., Kremser, J., Shi, H., Kreutz, C. and Al-Hashimi, H.M. (2019) Direct evidence for $(\mathrm{G}) \mathrm{O} 6 \cdots \mathrm{H} 2-\mathrm{N} 4(\mathrm{C})+$ hydrogen bonding in transient $\mathrm{G}($ syn)-C+ and G(syn)-m5C+ Hoogsteen base pairs in duplex DNA from cytosine amino nitrogen off-resonance $\mathrm{R} 1 \rho$ relaxation dispersion measurements. Journal of magnetic resonance, 308, 106589.

83. Lu, X.J. and Olson, W.K. (2003) 3DNA: a software package for the analysis, rebuilding and visualization of three-dimensional nucleic acid structures. Nucleic acids research, 31, 51085121.

84. Lee, C.T., Yang, W.T. and Parr, R.G. (1988) Development of the Colle-Salvetti CorrelationEnergy Formula into a Functional of the Electron-Density. Phys Rev B, 37, 785-789.

85. Binkley, J.S., Pople, J.A. and Hehre, W.J. (1980) Self-Consistent Molecular-Orbital Methods .21. Small Split-Valence Basis-Sets for 1st-Row Elements. Journal of the American Chemical Society, 102, 939-947.

86. Krishnan, R., Binkley, J.S., Seeger, R. and Pople, J.A. (1980) Self - consistent molecular orbital methods. XX. A basis set for correlated wave functions. The Journal of chemical physics, 72, 650-654.

87. Wolinski, K., Hinton, J.F. and Pulay, P. (1990) Efficient Implementation of the GaugeIndependent Atomic Orbital Method for Nmr Chemical-Shift Calculations. Journal of the American Chemical Society, 112, 8251-8260.

88. Chu, C.C., Liu, B., Plangger, R., Kreutz, C. and Al-Hashimi, H.M. (2019) m6A minimally impacts the structure, dynamics, and Rev ARM binding properties of HIV-1 RRE stem IIB. Plos One, 14, e0224850. 
89. Liu, B., Merriman, D.K., Choi, S.H., Schumacher, M.A., Plangger, R., Kreutz, C., Horner, S.M., Meyer, K.D. and Al-Hashimi, H.M. (2018) A potentially abundant junctional RNA motif stabilized by $\mathrm{m}(6) \mathrm{A}$ and $\mathrm{Mg}(2)$. Nat Commun, 9, 2761.

90. Merriman, D.K., Yuan, J., Shi, H., Majumdar, A., Herschlag, D. and Al-Hashimi, H.M. (2018) Increasing the length of poly-pyrimidine bulges broadens RNA conformational ensembles with minimal impact on stacking energetics. RNA, 24, 1363-1376.

91. Ippolito, J.A. and Steitz, T.A. (1998) A 1.3-A resolution crystal structure of the HIV-1 transactivation response region RNA stem reveals a metal ion-dependent bulge conformation. Proc Natl Acad Sci, 95, 9819-9824.

92. Fonville, J.M., Swart, M., Vokacova, Z., Sychrovsky, V., Sponer, J.E., Sponer, J., Hilbers, C.W., Bickelhaupt, F.M. and Wijmenga, S.S. (2012) Chemical shifts in nucleic acids studied by density functional theory calculations and comparison with experiment. Chemistry, 18, $12372-12387$.

93. Kumar, S., Mapa, K. and Maiti, S. (2014) Understanding the effect of locked nucleic acid and 2'-O-methyl modification on the hybridization thermodynamics of a miRNA-mRNA pair in the presence and absence of AfPiwi protein. Biochemistry, 53, 1607-1615.

94. Merriman, D.K., Xue, Y., Yang, S., Kimsey, I.J., Shakya, A., Clay, M. and Al-Hashimi, H.M. (2016) Shortening the HIV-1 TAR RNA Bulge by a Single Nucleotide Preserves Motional Modes over a Broad Range of Time Scales. Biochemistry, 55, 4445-4456.

95. Matera, A.G. and Wang, Z. (2014) A day in the life of the spliceosome. Nat Rev Mol Cell Biol, 15, 108-121.

96. Klinge, S. and Woolford, J.L., Jr. (2019) Ribosome assembly coming into focus. Nat Rev Mol Cell Biol, 20, 116-131.

97. Ganser, L.R., Chu, C.C., Bogerd, H.P., Kelly, M.L., Cullen, B.R. and Al-Hashimi, H.M. (2020) Probing RNA Conformational Equilibria within the Functional Cellular Context. Cell Rep, 30, 2472-2480 e2474.

98. Ganser, L.R., Kelly, M.L., Patwardhan, N.N., Hargrove, A.E. and Al-Hashimi, H.M. (2019) Demonstration that small molecules can bind and stabilize low-abundance short-lived RNA excited conformational states. $J \mathrm{Mol}$ Biol.

99. Chu, C.C., Plangger, R., Kreutz, C. and Al-Hashimi, H.M. (2019) Dynamic ensemble of HIV-1 RRE stem IIB reveals non-native conformations that disrupt the Rev-binding site. Nucleic Acids Res, 47, 7105-7117.

100. Leamy, K.A., Assmann, S.M., Mathews, D.H. and Bevilacqua, P.C. (2016) Bridging the gap between in vitro and in vivo RNA folding. $Q$ Rev Biophys, 49, e10.

101. Weeks, K.M. (2010) Advances in RNA structure analysis by chemical probing. Curr Opin Struct Biol, 20, 295-304.

102. Mustoe, A.M., Lama, N.N., Irving, P.S., Olson, S.W. and Weeks, K.M. (2019) RNA base-pairing complexity in living cells visualized by correlated chemical probing. Proc Natl Acad Sci, 116, 24574-24582.

103. Ge, J., Liu, H. and Yu, Y.T. (2010) Regulation of pre-mRNA splicing in Xenopus oocytes by targeted 2'-O-methylation. RNA, 16, 1078-1085. 


\section{TABLES AND FIGURES}

Table 1. Melting temperature and thermodynamic parameters derived from melting curves in the absence and presence of $\mathrm{Mg}^{2+}$.

\begin{tabular}{|c|c|c|c|c|c|c|c|c|}
\hline & \multicolumn{4}{|c|}{$\mathrm{No} \mathrm{Mg}^{2+}$} & \multicolumn{4}{|c|}{$3 \mathrm{mM} \mathrm{Mg}^{2+}$} \\
\hline Sample & $\mathrm{T}_{\mathrm{m}}\left({ }^{\circ} \mathrm{C}\right)$ & $\begin{array}{c}\Delta \mathrm{H}^{\circ} \\
(\mathrm{kcal} / \mathrm{mol})\end{array}$ & $\begin{array}{c}\mathrm{T} \Delta \mathrm{S}^{\circ} \\
(\mathrm{kcal} / \mathrm{mol})\end{array}$ & $\begin{array}{c}\Delta \mathrm{G}^{\circ}{ }_{37^{\circ} \mathrm{C}} \\
(\mathrm{kcal} / \mathrm{mol})\end{array}$ & $\mathrm{T}_{\mathrm{m}}\left({ }^{\circ} \mathrm{C}\right)$ & $\begin{array}{c}\Delta \mathrm{H}^{\circ} \\
(\mathrm{kcal} / \mathrm{mol})\end{array}$ & $\begin{array}{c}\mathrm{T} \Delta \mathrm{S}^{\circ} \\
(\mathrm{kcal} / \mathrm{mol})\end{array}$ & $\begin{array}{c}\Delta \mathrm{G}^{\circ}{ }_{37^{\circ} \mathrm{C}} \\
(\mathrm{kcal} / \mathrm{mol})\end{array}$ \\
\hline TAR & $65.2 \pm 0.3$ & $-62.9 \pm 1.3$ & $-57.7 \pm 1.2$ & $-5.2 \pm 0.1$ & $77.8 \pm 0.5$ & $-73.3 \pm 1.0$ & $-64.7 \pm 0.9$ & $-8.5 \pm 0.1$ \\
\hline TAR-A35 & $65.4 \pm 0.2$ & $-63.0 \pm 1.1$ & $-57.7 \pm 1.0$ & $-5.3 \pm 0.1$ & $77.6 \pm 0.3$ & $-71.3 \pm 2.2$ & $-63.1 \pm 2.0$ & $-8.3 \pm 0.2$ \\
\hline $\begin{array}{c}\text { TAR- } \\
\text { C24U25A35 }\end{array}$ & $65.5 \pm 0.2$ & $-65.0 \pm 3.0$ & $-59.5 \pm 2.7$ & $-5.5 \pm 0.2$ & $77.5 \pm 0.3$ & $-70.4 \pm 2.7$ & $-62.3 \pm 2.5$ & $-8.1 \pm 0.3$ \\
\hline
\end{tabular}

Experiments were run in triplicates (Methods) and the values with standard deviation are reported. $\Delta G^{\circ}{ }_{37^{\circ} \mathrm{C}}=\Delta \mathrm{G}^{\circ}$ (folded TAR) $-\Delta \mathrm{G}^{\circ}$ (single-stranded TAR). Experiments were performed at a ramp of 1 ${ }^{\circ} \mathrm{C} /$ min. Samples were $\sim 3.0-3.5 \mu \mathrm{M}$ in NMR buffer ( $15 \mathrm{mM} \mathrm{NaPO}_{4}, 25 \mathrm{mM} \mathrm{NaCl}$ and $0.1 \mathrm{mM}$ EDTA) at $\mathrm{pH} 6.4$. 
A<smiles>COC1C(O)C(CO)OC1C(C)(C)C</smiles>

Base $=$ Cytosine $(C)$,

Adenine (A), Guanine (G), Uracil (U)

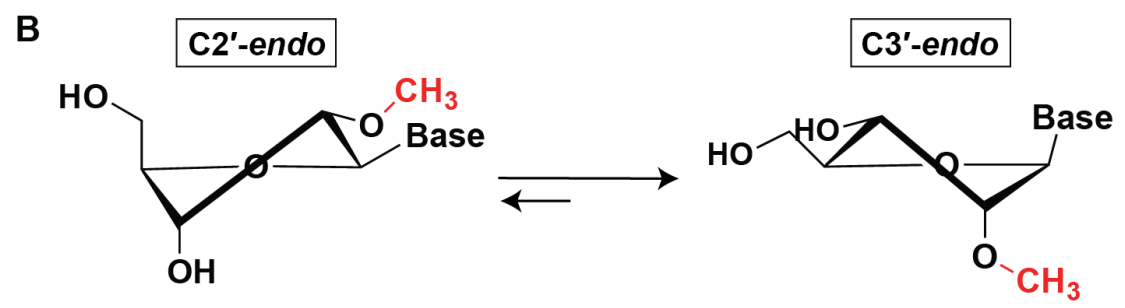

C
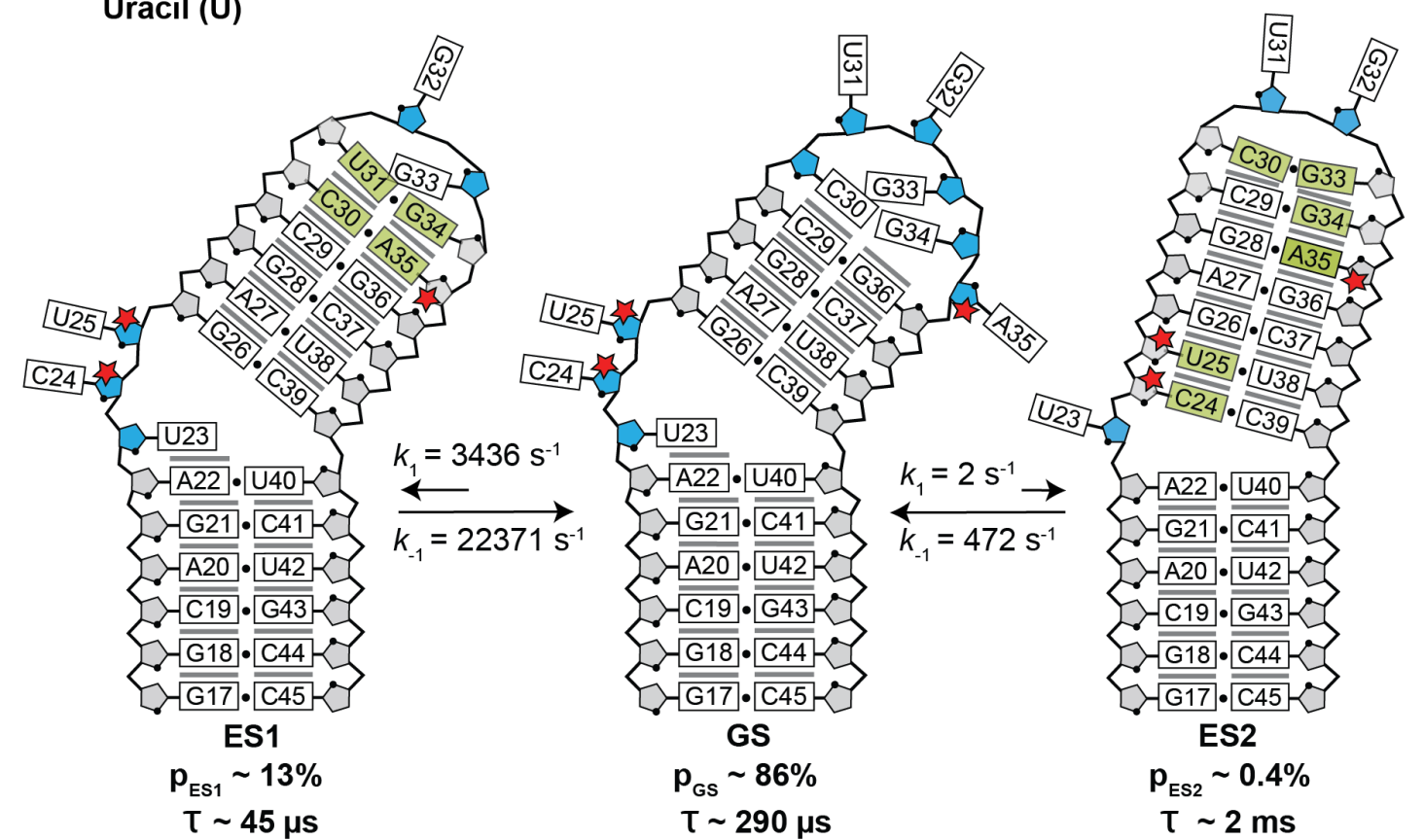

Figure 1. A) Chemical structure of a 2'-O-methylated nucleoside ( $\mathrm{Nm})$. B) $\mathrm{Nm}$ biases the sugar pucker towards C3'-endo (34). C) Secondary structure of the TAR GS and ESs. The exchange parameters shown are for TAR in the absence of $\mathrm{Mg}^{2+}$ as reported previously $(66,67)$. Nucleotides that undergo sugar repuckering from C2'-endo (blue) in the GS to C3'-endo (grey) in the ES are indicated using green bases in the ESs. Red stars indicate the Nm-modified residues. Black dots denote base pairing and grey lines denote stacking. The lifetime $(\tau)$ of each ES is equal to $1 / k_{-1}$, and $\tau$ of the GS $=1 /\left(k_{1, E S 1}+\right.$

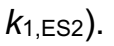



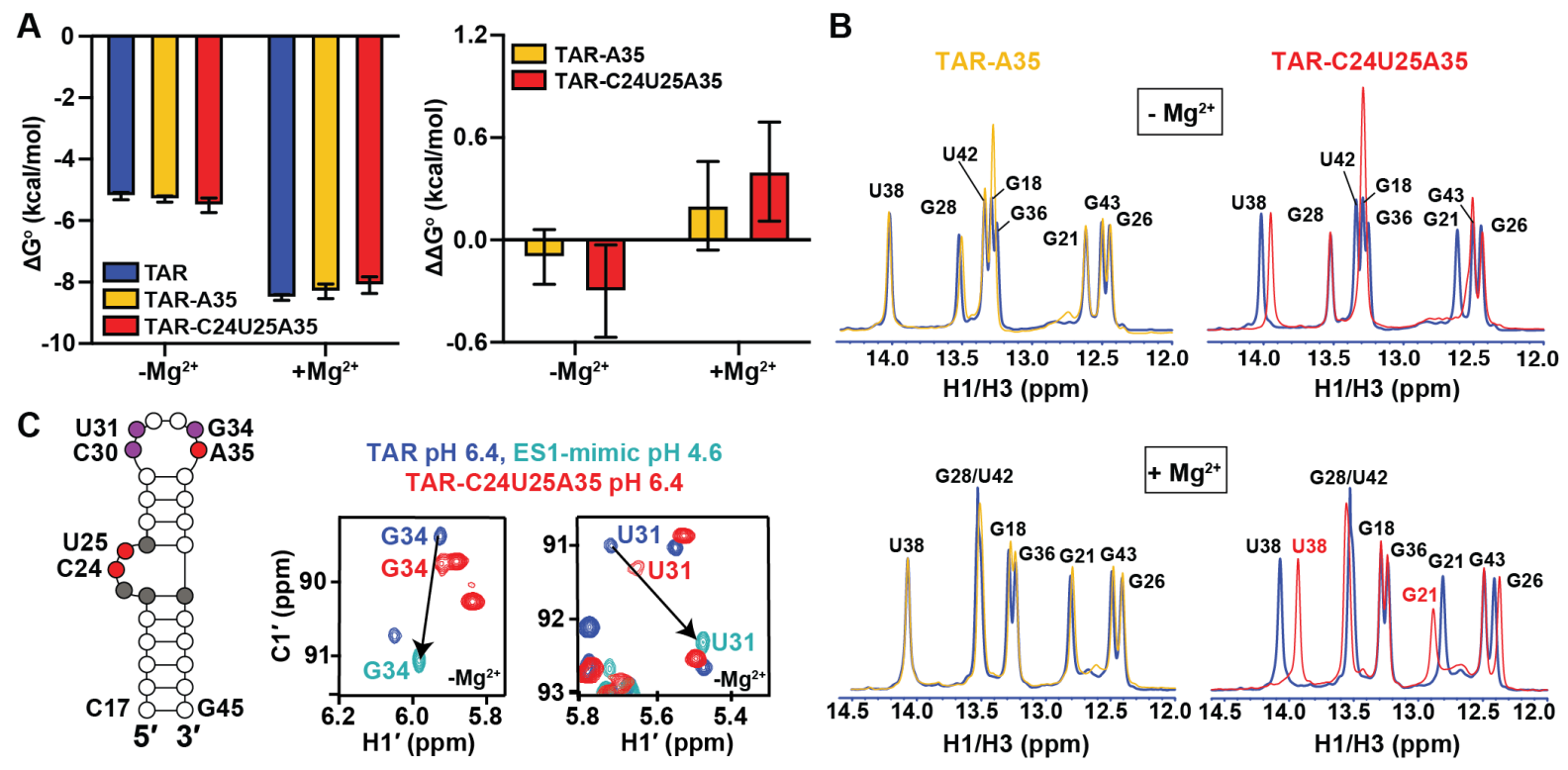

Figure 2. Impact of $\mathrm{Nm}$ on the thermal stability of TAR GS. A) $\Delta \mathrm{G}^{\circ}$ and $\Delta \Delta \mathrm{G}^{\circ}$ values derived from melting curves in the absence and presence of $3 \mathrm{mM} \mathrm{Mg}^{2+}$. Error bars on $\Delta \mathrm{G}^{\circ}$ denote standard deviation of triplicate measurements as described in the methods. Error bars on $\Delta \Delta G^{\circ}$ were obtained by propagating the errors from triplicate measurements (see Methods). B) Overlays comparing $1 \mathrm{D}^{1} \mathrm{H}$ imino spectra for unmodified TAR (blue), TAR-A35 (yellow) and TAR-C24U25A35 (red) in the absence and presence of $3 \mathrm{mM} \mathrm{Mg}^{2+}$. All samples were unlabeled. C) Secondary structure of TAR with Nmmodified residues shown in red, residues showing CSPs towards ES1 are in purple, and residues exhibiting CSPs due to bulge modifications are in grey. Overlay of natural abundance $2 \mathrm{D}\left[{ }^{13} \mathrm{C},{ }^{1} \mathrm{H}\right]$ HSQC spectra for the C1'-H1' region in TAR (pH 6.4), TAR ES1-mimic (pH 4.6) (66), and TARC24U25A35 (pH 6.4), in the absence of $\mathrm{Mg}^{2+}$. Lowering the $\mathrm{pH}$ to 4.6 shifts the population of TAR (structure shown in Figure S2) to stabilize ES1 as the dominant conformation due to protonation of the A35-C30 mismatch unique to ES1 (66). Spectra with complete assignments are shown in Figures S1 and S2. 

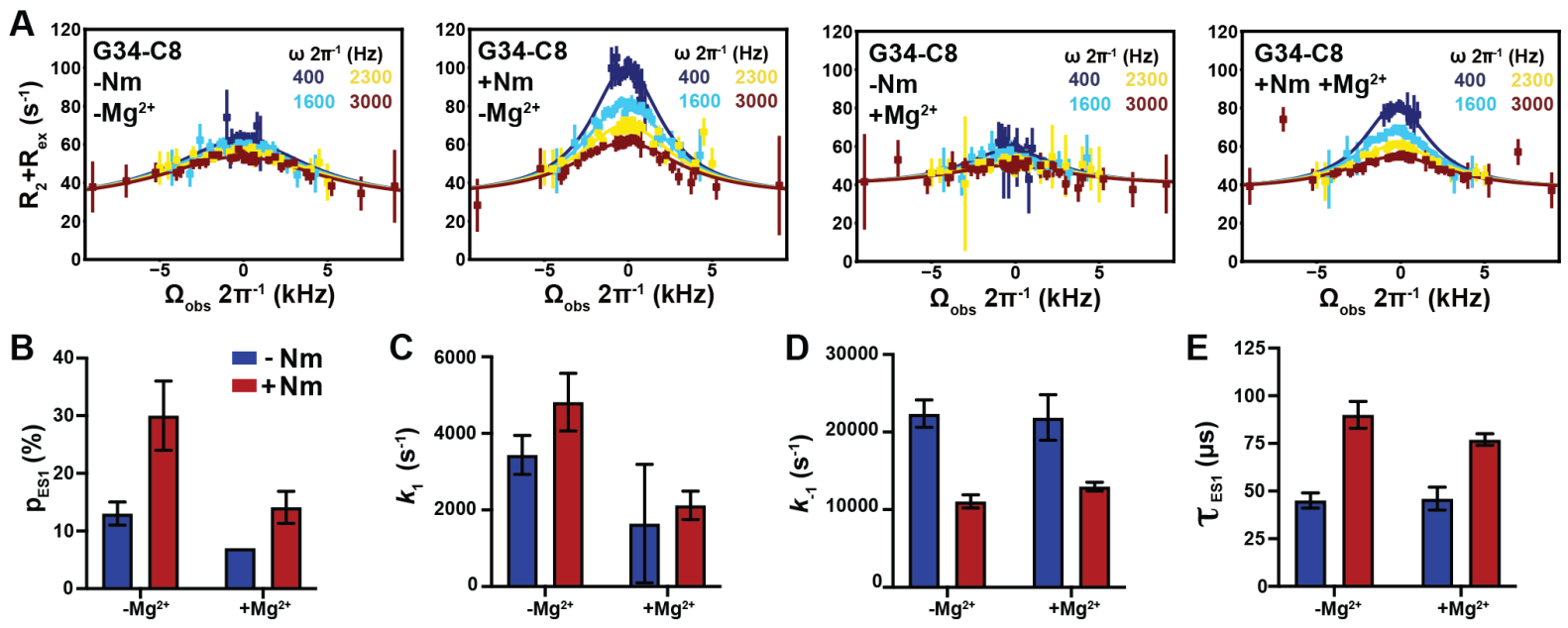

Figure 3. Impact of $\mathrm{Nm}$ on GS-ES1 exchange. A) Off-resonance $R_{1 \rho}{ }^{13} \mathrm{C}$ RD profiles for G34-C8 in TAR and TAR-C24U25A35 at $25^{\circ} \mathrm{C}$, in the absence and presence of $1 \mathrm{mM} \mathrm{Mg}^{2+} . R_{1 \rho}$ data was fit with a two-state model using Bloch-McConnell equations. Spin-lock powers are color-coded. Errors in the $R_{1 \rho}$ data were calculated as described previously (63). B-E) Comparison of ES1 population (pES1), forward $\left(k_{1}\right)$ and backward $\left(k_{-1}\right)$ rate constants, and lifetime $(\tau)$ between TAR and TAR-C24U25A35, in the absence and presence of $1 \mathrm{mM} \mathrm{Mg}^{2+}$, as obtained from fitting $R_{1 \rho}$ data. Error bars were calculated using a Monte-Carlo scheme (63). 

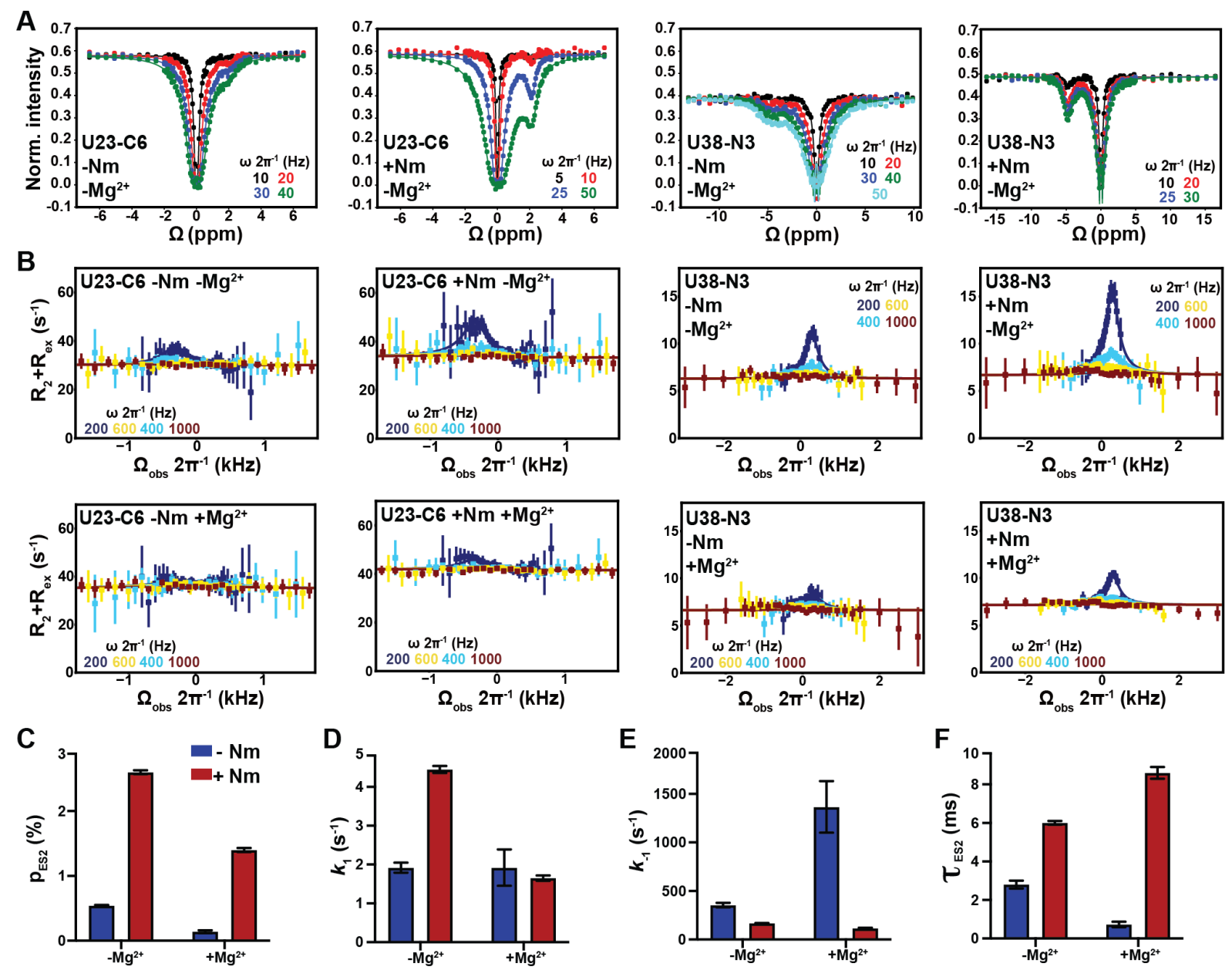

Figure 4. Impact of Nm on GS-ES2 exchange in TAR. A) ${ }^{13} \mathrm{C}$ and ${ }^{15} \mathrm{~N}$ CEST profiles in TAR and TARC24U25A35 in the absence of $\mathrm{Mg}^{2+}$. B) Off-resonance ${ }^{13} \mathrm{C}$ and ${ }^{15} \mathrm{~N} R_{1 p} \mathrm{RD}$ profiles for U23-C6 and U38-N3 in TAR and TAR-C24U25A35 in the absence and presence of $1 \mathrm{mM}$ of $\mathrm{Mg}^{2+} . R_{1 p}$ data was fit with a two-state model using Bloch-McConnell equations. Fits of $R_{1 \rho}$ profiles were preformed fixing the population to the value measured using CEST. Errors in the $R_{1 \rho}$ data were calculated as described previously (63). Spin-lock powers in CEST and $R_{1 \rho}$ are color-coded. C-F) Comparison of $\mathrm{p}_{\mathrm{ES} 2}, k_{1}$ and $k-1$ rate constants, and $\tau$ ES2 between TAR and TAR-C24U25A35 in the absence and presence of $1 \mathrm{mM}$ $\mathrm{Mg}^{2+}$ obtained from fitting the CEST data. Error bars were calculated using a Monte-Carlo scheme (55). 

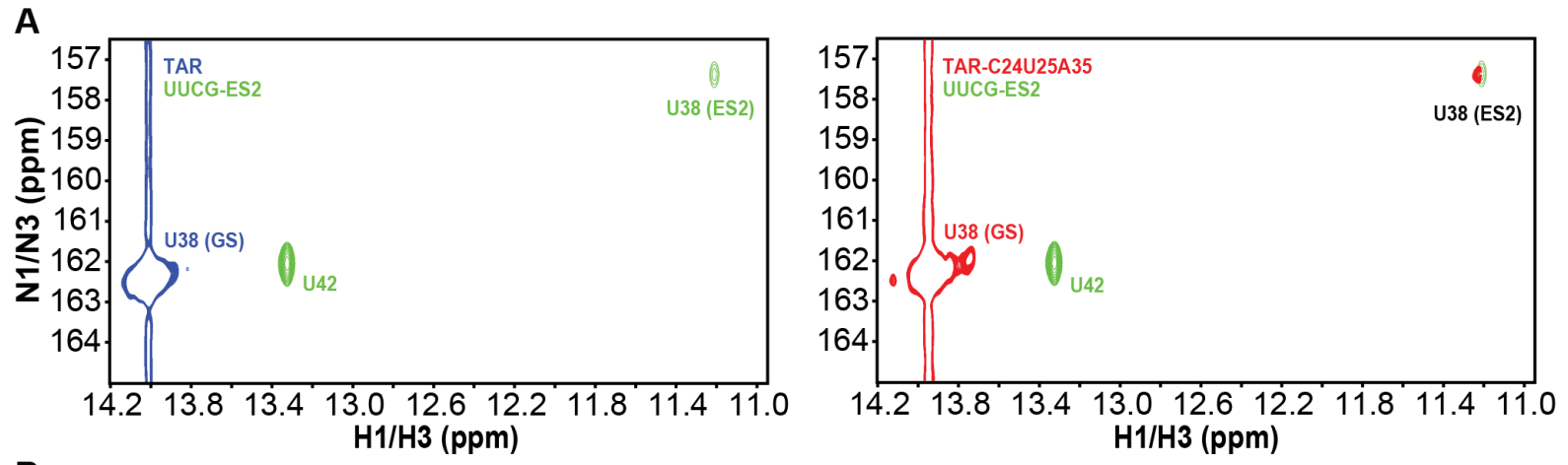

B
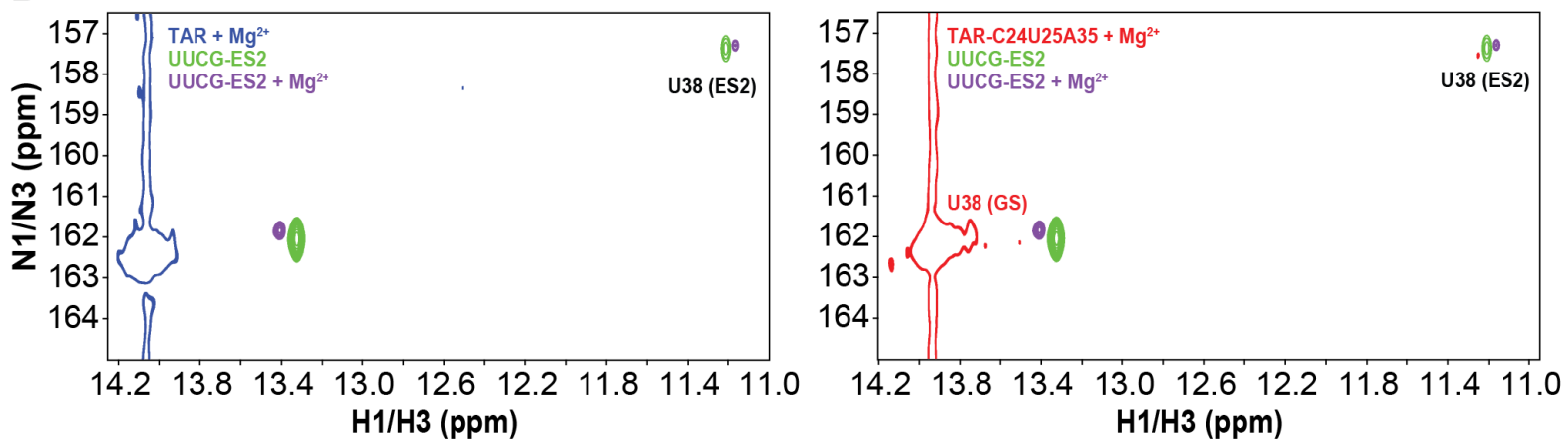

Figure 5. Direct observation of ES2 U38-N3H3 resonance in the Nm-modified TAR sample in NMR 2D spectra. Overlay of 2D $\left[{ }^{1} \mathrm{H},{ }^{15} \mathrm{~N}\right] \mathrm{HSQC}$ spectra of uniformly ${ }^{15} \mathrm{~N} /{ }^{13} \mathrm{C}$ labeled UUCG-ES2 (green/purple) with ${ }^{15} \mathrm{~N}(\mathrm{U} 38)$ site-labeled TAR (blue) and TAR-C24U25A35 (red) in the A) absence and B) presence of $1 \mathrm{mM} \mathrm{Mg}^{2+}$. The ES2 U38-N3H3 resonance is absent in unmodified TAR and clearly visible in TARC24U25A35. Full $\left[{ }^{15} \mathrm{~N},{ }^{1} \mathrm{H}\right] 2 \mathrm{D}$ HSQC spectra of UUCG-ES2 are shown in Figure S7.

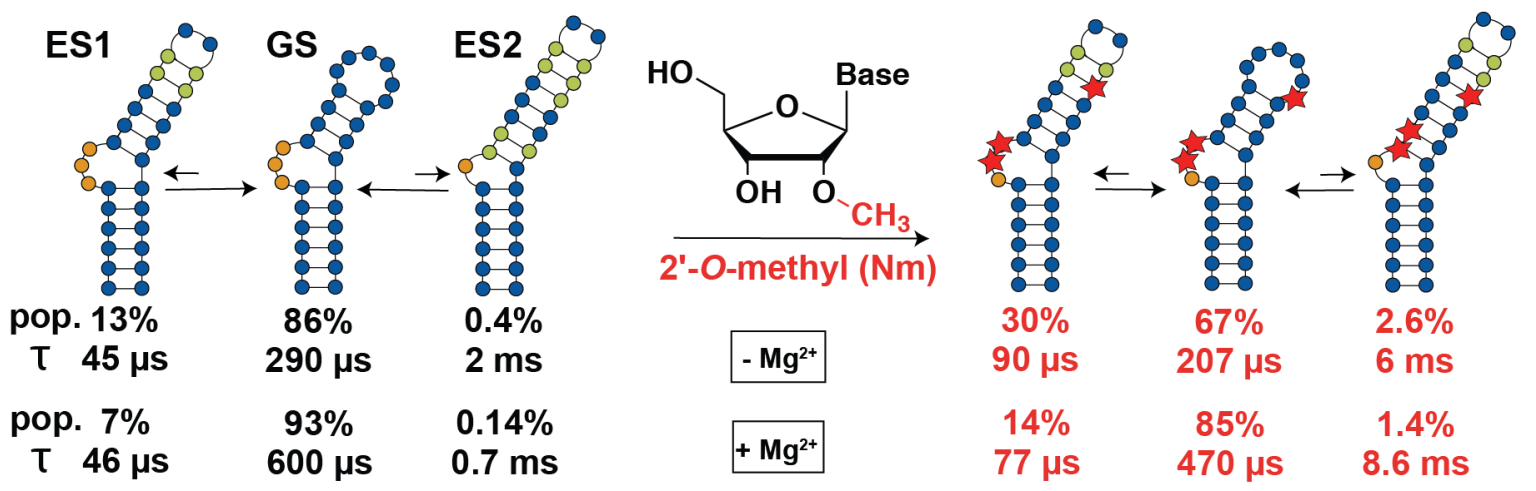

Figure 6. Summary of the effect of $\mathrm{Nm}$ on GS-ES exchange in the presence and absence of $\mathrm{Mg}^{2+}$. Bulge nucleotides are shown in orange. Nucleotides that transition from being unpaired in GS to paired in the ES are shown in green. Red stars indicate the Nm-modified residues. Pop and $\tau$ refer to the population and lifetime of the conformational state (GS, ES1 or ES2). 